Research Article

\title{
Chaotic Behaviour and Bifurcation in Real Dynamics of Two- Parameter Family of Functions including Logarithmic Map
}

\author{
Mohammad Sajid \\ Mechanical Engineering Department, College of Engineering, Qassim University, Buraidah, Al Qassim, Saudi Arabia \\ Correspondence should be addressed to Mohammad Sajid; msajd@qu.edu.sa
}

Received 2 January 2020; Revised 24 May 2020; Accepted 9 June 2020; Published 7 July 2020

Academic Editor: Alfred Peris

Copyright (C) 2020 Mohammad Sajid. This is an open access article distributed under the Creative Commons Attribution License, which permits unrestricted use, distribution, and reproduction in any medium, provided the original work is properly cited.

\begin{abstract}
The focus of this research work is to obtain the chaotic behaviour and bifurcation in the real dynamics of a newly proposed family of functions $f_{\lambda, a}(x)=x+(1-\lambda x) \ln a x ; x>0$, depending on two parameters in one dimension, where assume that $\lambda$ is a continuous positive real parameter and $a$ is a discrete positive real parameter. This proposed family of functions is different from the existing families of functions in previous works which exhibits chaotic behaviour. Further, the dynamical properties of this family are analyzed theoretically and numerically as well as graphically. The real fixed points of functions $f_{\lambda, a}(x)$ are theoretically simulated, and the real periodic points are numerically computed. The stability of these fixed points and periodic points is discussed. By varying parameter values, the plots of bifurcation diagrams for the real dynamics of $f_{\lambda, a}(x)$ are shown. The existence of chaos in the dynamics of $f_{\lambda, a}(x)$ is explored by looking period-doubling in the bifurcation diagram, and chaos is to be quantified by determining positive Lyapunov exponents.
\end{abstract}

\section{Introduction}

During the past four decades, there are significantly developments in computational power, advances in technology, and mathematical theory that have facilitated formulation of nonlinear approaches for intricate systems $[1,2]$ since many physical, socioeconomic, and natural systems are intrinsically nonlinear, so these systems show large range of characteristics. During the same time, chaos has been also noted in several experimental works and it redesigns many researches in different fields of engineering and science [3-5]; also, the study of chaotic behaviour in dynamical systems has been the interest of many scientists, engineers, and mathematicians. Applications of it can be extensively found in variety of disciplines such as modeling [6,7], optimization [8], stock market [9], photovoltaic plant [10], fashion cycle model [11], and other [5]. The global dynamical analysis of an $\mathrm{H}$-bridge parallel resonant converter under a zero current switching control is described in [12]. The dynamics of a DC-AC resonant self-oscillating LC series inverter is analyzed in [13] from the point of view of piecewise smooth dynamical systems, and the bifurcation analysis is performed in a one- dimensional parameter space. In [14], a discrete dynamics approach is applied to show various tricks on using the sparse computing method to ontology learning and verify its efficiency through experiments. The recurrence in nonautonomous discrete dynamical systems is studied in [15]. A bifurcation analysis of gender equality and fertility is illustrated in [16]; it is shown how bifurcation theory can be used to study the process of increasing gender equality and its implications on fertility. In [17], the discrete time neoclassical one-sector growth model with differential savings is discussed while assuming Kadiyala production function which shows a variable elasticity of substitution symmetric with respect to capital and labor. A nonlinear version of the efficiency-wage competition model pioneered by Hahn (1987) is developed in [18], and the chaotic behaviour is also seen in the parameter region.

Appearance of chaos in systems is combined presence of nonlinear interdependence, order and determinism, and sensitive dependence. The word chaos is derived from the ancient Greek word Xáo ; normally, it means a state lacking order or predictability. Chaos is a fascinating mathematical and physical phenomenon. The study of chaos shows that 
simple systems can exhibit complex and unpredictable behaviour $[19,20]$. Generally, chaos can be defined in many different forms according to conditions, observations, or applications of the object. As a notion in the mathematical sense, the chaos is developed about 1980. After that, the following characteristic became common opinion: a deterministic evolution is chaotic if (i) this shows property of sensitivity to initial condition and (ii) it consists a nonlinear mechanism confirming the mixing of trajectories and these return back in a bounded domain of the phase space.

The phenomenon of chaos is generally related to the field known as dynamical systems [21], and it can be characterized in the dynamics by sensitive dependence on the initial conditions. Dynamical system is the study of the system or processes that change over time. Often, investigations in dynamical systems hold many surprises and show the relationships between order and disorder, simplicity and complexity $[19,22]$. The main purpose to study a dynamical system is to observe the asymptotic behaviour of the trajectories that corresponds to long-term observations. In other words, we can say which initial conditions have gone to identical long-term behaviour. And which ones lead to dramatically different behaviour? One of the main things in dynamical systems is the study of the behaviour of orbits near fixed points. The fixed point is said to be stable if the system tends to come back to it after a little disturbance and is said to be unstable if the initial discrepancy goes to grow.

Moreover, the importance of the real dynamics of functions can be observed in the dynamics of functions in the complex plane and often such type of investigations describe Fatou sets, Julia sets, and some other dynamical results in the complex plane [23-27] and references therein.

1.1. Definitions and Basic Concepts. The mathematical models for the above types of applications are normally formulated in the forms of difference and differential equations. To study solution of these types of equations, generally, iterations are applicable. For a given initial value $x_{0}$ in one dimensional map, we compute iterations as $x_{1}=f\left(x_{0}\right), x_{2}=$ $f\left(x_{1}\right), x_{3}=f\left(x_{2}\right), \cdots$. The sequence $x_{0}, x_{1}, x_{2}, x_{3}, \cdots$ defines the trajectory of the dynamical variable $x_{n}$, where the time $n$ is discrete. Points which come back to the same value after a finite number of iterations of function are called periodic points, and a periodic point with period equal to one is known as a fixed point. For a periodic point $x_{0}$ of period $p$, the orbit $x_{0}, x_{1}=f\left(x_{0}\right), x_{2}=f^{2}\left(x_{0}\right), \cdots, x_{p-1}=f^{p-1}\left(x_{0}\right)$ is called a cycle or a periodic cycle. The periodic point $x_{0}$ of period $p$ is classified as follows. If $\left|\left(f^{p}\right)^{\prime}\left(x_{0}\right)\right|<1$, then the periodic point $x_{0}$ is known as attracting. If $\left|\left(f^{p}\right)^{\prime}\left(x_{0}\right)\right|>1$, then the periodic point $x_{0}$ is known as repelling. If $\mid\left(f^{p}\right)^{\prime}$ $\left(x_{0}\right) \mid=1$, then the periodic point $x_{0}$ is known as neutral (rationally or irrationally indifferent). A fixed point $x_{0}$ of $f(x)$ is called attracting, neutral (indifferent), or repelling if $\left|f^{\prime}\left(x_{0}\right)\right|<1,\left|f^{\prime}\left(x_{0}\right)\right|=1$, or $\left|f^{\prime}\left(x_{0}\right)\right|>1$, respectively.

As a parameter varies, qualitative changes in the behaviour of the system can occur at certain parameter values. Each of such a change is known as a bifurcation. In other words, when the parameter crosses through the certain value, then the dynamics of the function changes. One method displaying the points, at which a parametrized family of functions bifurcates, is called a bifurcation diagram. The bifurcation diagram is designed to show eventual behaviour of iterates, such as convergence or periodicity or unpredictability.

In the dynamics of functions, chaos is generally designated by the sensitive dependence on the initial conditions. Several techniques are available to identify as well as quantify the chaotic behaviour in the dynamics. It can identify by looking period-doubling in the bifurcation diagram or observing time series behaviour. A positive value of the Lyapunov exponent proves that chaotic behaviour exists in the dynamical system $[28,29]$. For a trajectory $\left\{x_{k}\right\}, k=$ $0,1,2, \cdots$ starting from $x_{0}$, the Lyapunov exponent of $f(x)$ is defined as

$$
L=\lim _{j \rightarrow \infty} \frac{1}{j} \sum_{k=0}^{j-1} \ln \left|f^{\prime}\left(x_{k}\right)\right| .
$$

1.2. Previous Works. Using powerful computation through the computer, many research works have been involved in the field of dynamical systems associated with chaos. For discrete time systems, maps are easy and fast to simulate on the computer. Recently, many developments have been happened in theoretical studies which can be seen in [30]. Specific periodic motions in the pendulum are predicted semianalytically and analytical bifurcation trees of periodic motions to chaos are determined by using the discrete maps [31]. The following three discrete models of population dynamics are described in [32]: (i) the classical delay logistic equation, (ii) its variant which incorporates a harvesting rate, and (iii) the PerezMalta-Coutinho equation.

A one-parameter family of maps which unfolds a Landen-type map obtained by Boros and Moll is considered in [33], and it described the Neimark-Sacker bifurcations that appear in the route towards the parameter value that corresponds to this map. Neimark-Sacker bifurcation and controlling chaos in a two-dimensional discrete-time predator-prey system are investigated in [34]. The stability of the fixed point of the model and the existence conditions of the Neimark-Sacker bifurcation are also studied. The dynamics of a family of one-dimensional linear-power maps is found in [35], and cascades of alternating smooth bifurcations and border collision bifurcations with singularity are described there.

For a large set of parameter values, the cobweb diagram exhibits observable chaos by using numerical simulation [36]. A specific sequence of bifurcations gives a route to chaos. The following are three evidences of typical routes for dissipative systems: (i) the period-doubling route, (ii) the intermittency, and (iii) the Ruelle-Takens bifurcation [30]. For a family of one-dimensional maps corresponding to Fibonacci-generating functions, the chaotic behaviour in the dynamics is described [37]. For a family of onedimensional transcendental functions, the real dynamics is studied in $[38,39]$. For families of transcendental functions depending on two parameters, the bifurcation as well as chaotic behaviour in the real dynamics is investigated in $[38,40]$ 


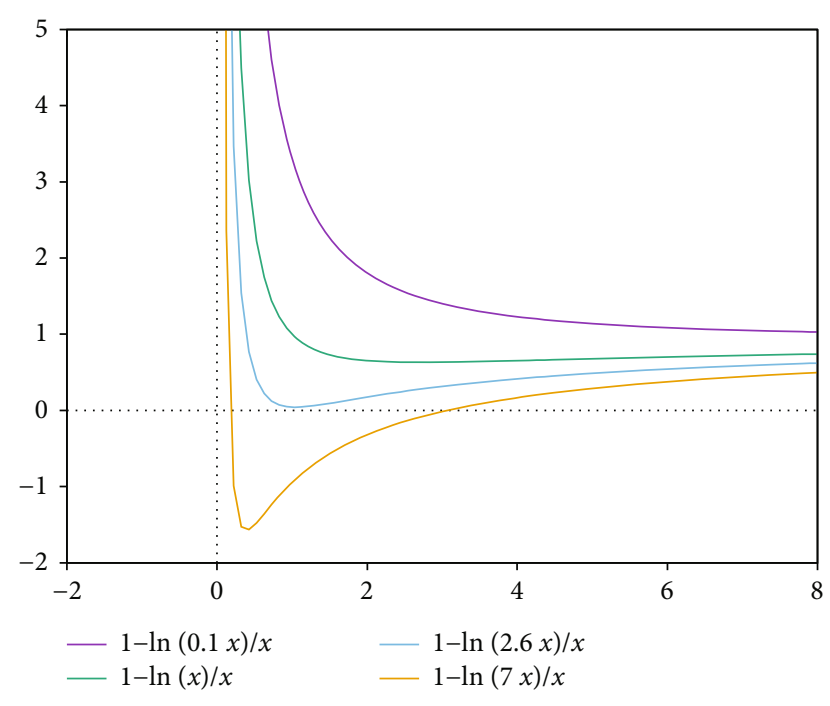

FIgURE 1: The graph of $\zeta(x)=1-(\ln a x / x)$ for $a=0.1,1,2.6,7$.

and the analysis of the real dynamics of iterative methods is found in [41]. The real dynamics of $\left(b^{x}-1\right) / x$ associated with one parameter is explained in [42]. For two-parameter families of special type of generating functions, the bifurcation as well as chaos in the real dynamics is described by author $[43,44]$. The description of the real dynamics of transcendental functions is essential in many phenomena. For instance, the dynamics (iteration) associated with Newton's method is as the root finder.

Sometimes, the discrete systems become more important when, for the ODE systems, some complex models were transformed into discrete models, namely, the Poincaré map. Such Poincaré map, as a discrete system, is an important tool to analyze the dynamics of the continuous-time systems, for example, the biped robots, as complex systems, where the determination of discrete system is important to analyze the motion. See the following associated works on these themes: (i) design of an explicit expression of the Poincaré map for the passive dynamic walking of the compass-gait biped model [45]; (ii) walking dynamics of the passive compass-gait model under OGY-based statefeedback control: (a) rise of the Neimark-Sacker bifurcation [46], (b) emergence of bifurcations and chaos [47], and (c) analysis of local bifurcations via the hybrid Poincaré map [48]; and (iii) calculation of the Lyapunov exponents in the compass-gait model under OGY control via a hybrid Poincaré map [49].

1.3. Organization of the Present Work. The dynamics of twoparameter family of functions changes when both values of parameters cross through certain values. We can observe these changes in the dynamics by the bifurcation diagram. In the real dynamics, the period-doubling in the bifurcation diagram shows route of chaos. Consider a discrete dynamical system in the present paper which is obtained by iteration of function $f_{\lambda, a}(x)$, depending on real parameters $\lambda$ and $\mu$. For computation of the bifurcation points, we have to solve the equations associated to fixed points and neutral fixed point condition, i.e., solve the following equations $f_{\lambda, a}^{p}(x)=x$ and $\left|\left(f_{\lambda, a}^{p}\right)^{\prime}(x)\right|=1$.

The organization of this paper is as follows. The real fixed points of $f_{\lambda}(x)$ with their nature on the real line are described in Section 2. The real periodic points of $f_{\lambda}(x)$ of period more than or equal to 2 are numerically discussed in Section 4 . Further, in Section 4, the real periodic points of $f_{\lambda}(x)$ of period three are explained. Furthermore, the bifurcation and chaos in the dynamics are explored in Section 5. At the end, in Section 6 , conclusions are mentioned.

\section{Real Fixed Points and Periodic Points of $f_{\lambda}$ $\in \mathscr{F}$ with Their Nature}

Generally, the dynamics of polynomials as well as rational functions is somewhat simpler than transcendental functions. The present research work deals with the investigation of the dynamical properties of a newly proposed family of transcendental functions which depends on two parameters. Let

$$
\mathscr{F}=\left\{f_{\lambda, a}(x)=x+(1-\lambda x) \ln a x: x>0, \lambda>0, a>0\right\}
$$

be a family of transcendental functions depending on two parameters in one dimension associated with logarithmic map. In this study, assume that $\lambda$ is a continuous parameter and $a$ is a discrete parameter. This proposed family of functions is different from the existing families of functions in previous research works which exhibits chaotic behaviour.

In this section, we focus on the real fixed points as well as the real periodic points of the functions $f_{\lambda} \in \mathscr{F}$. The following lemma is needed in the sequel:

Lemma 1. Suppose $\zeta(x)=1-(\ln a x / x), x \in \mathbb{R}^{+} \backslash\{0\}, a>0$. Then, the function $\zeta(x)$ attains local minima at $x=e / a$. Its local minimum value is $1-(a / e)$ and $\zeta\left(x^{*}\right)=-1$, where $x^{*}$ is a solution of $e^{2 x}-a x=0$. Moreover, $\zeta(x) \longrightarrow+\infty$ as $x \longrightarrow 0^{+}$and $\zeta(x) \longrightarrow 1$ as $x \longrightarrow+\infty$.

Proof. We have $\zeta^{\prime}(x)=(-1+\ln a x) / x^{2}$. For extrema, $\zeta^{\prime}(x)$ $=0$, then $x=e / a$. Since $\zeta^{\prime \prime}(x)=(3-2 \ln a x) / x^{3}$, then $\zeta^{\prime \prime}(e /$ $a)=\left(a^{3} / e^{3}\right)>0$ for $a>0$. Hence, $\zeta(x)$ attains local minima at $x=e / a$ if $a>0$. The local minimum value of $\zeta(e / a)$ is given as $1-(a / e)$. We can easily see the rest of the part in Figure 1 .

For real fixed points of $f_{\lambda, a}(x)$, we have $f_{\lambda, a}(x)=x$. It gives us $x+(1-\lambda x) \ln a x=x$. Then, it provides two real fixed points $x_{\lambda}=1 / \lambda$ and $x_{a}=1 / a$. For fix $a$ positive, the nature of the fixed points $x_{\lambda}$ and $x_{a}$ of $f_{\lambda, a}(x)$ is described as follows:

$$
\begin{aligned}
& f_{\lambda, a}^{\prime}(x)=1+\frac{1}{x}-\lambda(1+\ln a x), \\
& f_{\lambda, a}^{\prime}\left(x_{\lambda}\right)=1-\frac{\ln a x_{\lambda}}{x_{\lambda}}, \\
& f_{\lambda, a}^{\prime}\left(x_{a}\right)=1-\lambda+\frac{1}{x_{a}} .
\end{aligned}
$$


(i) For $a=1$, we define $\lambda_{1}=1$. We have $f_{\lambda, 1}^{\prime}\left(x_{\lambda}\right)=1$ $-\left(\ln x_{\lambda} / x_{\lambda}\right)$. Using Lemma 1 , we get $f_{\lambda, 1}^{\prime}\left(x_{\lambda}\right)>1$ for $0<\lambda<\lambda_{1}, f_{1,1}^{\prime}\left(x_{\lambda}\right)=1$ for $\lambda=\lambda_{1}$, and $0<f_{\lambda, 1}^{\prime}$ $\left(x_{\lambda}\right)<1$ for $\lambda>\lambda_{1}$. Hence, the fixed point $x_{\lambda}$ of $f_{\lambda, 1}(x)$ is repelling for $0<\lambda<\lambda_{1}$, rationally indifferent for $\lambda=\lambda_{1}$, and attracting for $\lambda>\lambda_{1}$

(ii) For $a=7$, we define $\lambda_{2}=1 / a$ and $\lambda_{3}=1 / x^{*}$, where $x^{*}$ is a solution of the equation $e^{2 x}-7 x=0$. We have $f_{\lambda, 7}^{\prime}\left(x_{\lambda}\right)=1-\ln 7 x_{\lambda} / x_{\lambda}$. Using Lemma 1 , we get $f_{\lambda, 7}^{\prime}$ $\left(x_{\lambda}\right)>1$ for $\lambda>\lambda_{2}$. Therefore, the fixed point $x_{\lambda}$ of $f_{\lambda, 7}(x)$ is repelling for $\lambda>\lambda_{2}$. Further, $f_{\lambda, 7}^{\prime}\left(x_{\lambda}\right)=1$ for $\lambda=\lambda_{2}$. Consequently, the fixed point $x_{\lambda}$ of $f_{\lambda, 7}(x)$ is rationally indifferent for $\lambda=\lambda_{2}$. Furthermore, $-1<f_{\lambda, 7}^{\prime}\left(x_{\lambda}\right)<1$ for $\lambda_{3}<\lambda<\lambda_{2}$. It follows that $\left|f_{\lambda, 7}^{\prime}\left(x_{\lambda}\right)\right|<1$ for $\lambda_{3}<\lambda<\lambda_{2}$. Hence, the fixed point $x_{\lambda}$ of $f_{\lambda, 7}(x)$ is attracting for $\lambda_{3}<\lambda<\lambda_{2}$. Next, $f_{\lambda, 7}^{\prime}\left(x_{\lambda}\right)=-1$ for $\lambda=\lambda_{3}$ by Lemma 1 . It gives that $\left|f_{\lambda, 7}^{\prime}\left(x_{\lambda}\right)\right|=1$ for $\lambda=\lambda_{3}$. Consequently, the fixed point $x_{\lambda}$ of $f_{\lambda, 7}(x)$ is rationally indifferent for $\lambda=\lambda_{3}$. Moreover, $f_{\lambda, 7}^{\prime}\left(x_{\lambda}\right)<-1$ for $\lambda<\lambda_{3}$ by Lemma 1 . It shows that $\left|f_{\lambda, 7}^{\prime}\left(x_{\lambda}\right)\right|>1$ for $\lambda<\lambda_{3}$. Therefore, the fixed point $x_{\lambda}$ of $f_{\lambda, 7}(x)$ is repelling for $\lambda<\lambda_{3}$

\section{Numerical Simulation of Real Cycles of Periods 2, 4, 8, and 16 with Their Nature}

The real periodic points of $f_{\lambda, a} \in \mathscr{F}$ are discussed here with their nature. The periodic points of $f_{\lambda, a}(x)$ are roots of $f_{\lambda, a}^{k}(x)=x$, i.e., $f_{\lambda, a}^{k-1}(x)+\left(1-\lambda f_{\lambda, a}^{k-1}(x)\right) \ln \left(a f_{\lambda, a}^{k-1}(x)\right)=x$. The analytical calculation of these periodic points of $f_{\lambda, a}(x)$ is significantly difficult and complicated to be obtained. When parameter $\lambda$ increases beyond certain value, then the function $f_{\lambda, a}(x)$ exhibits periodic points of period more than or equal to 2 . For $a=0.1,0.2,0.3,0.9,1,1.1$, the real cycles of periods $2,4,8$, and 16 with their stability are discussed by choosing certain values of parameter $\lambda$.

For $a=0.1$, the periodic points of periods $2,4,8$, and 16 of $f_{\lambda, a}(x)$ are numerically calculated at the following values of parameter $\lambda=2.4,2.55,2.58,2.592$, respectively:

(i) If $\lambda=2.4$, then the periodic points of 2-cycle of $f_{\lambda, a}$ ( $x$ ) are obtained as $\alpha_{1}=5.48280$ and $\alpha_{2}=12.78982$. It follows that

$$
\begin{aligned}
\left|f_{\lambda, a}^{\prime}\left(\alpha_{1}\right) f_{\lambda, a}^{\prime}\left(\alpha_{2}\right)\right| & =|0.22471 \times(-1.91237)| \\
& \approx 0.42973<1 .
\end{aligned}
$$

Therefore, the periodic 2-cycle of $f_{2.4,0.1}(x)$ is attracting

(ii) If $\lambda=2.55$, then the periodic points of 4-cycle of $f_{\lambda, a}(x)$ are found as $\alpha_{1}=3.89824, \alpha_{2}=12.32074, \alpha_{3}$ $=5.97255$, and $\alpha_{4}=13.30685$. It gives that

$$
\begin{aligned}
& \left|f_{\lambda, a}^{\prime}\left(\alpha_{1}\right) f_{\lambda, a}^{\prime}\left(\alpha_{2}\right) f_{\lambda, a}^{\prime}\left(\alpha_{3}\right) f_{\lambda, a}^{\prime}\left(\alpha_{4}\right)\right| \\
& =\mid 1.10878 \times(-2.00102) \\
& \quad \times(-0.06827) \times(-2.20337) \mid \approx 0.33375<1 .
\end{aligned}
$$

Hence, the periodic 4-cycle of $f_{2.55,0.1}(x)$ is attracting

(iii) If $\lambda=2.58$, then the periodic points of 8 -cycle of $f_{\lambda, a}(x)$ are determined as $\alpha_{1}=3.81940, \alpha_{2}=$ $12.34135, \alpha_{3}=5.85338, \alpha_{4}=13.40578, \alpha_{5}=3.56143$, $\alpha_{6}=12.01541, \alpha_{7}=6.50730$, and $\alpha_{8}=13.29114$. It follows that

$$
\begin{aligned}
\mid f_{\lambda, a}^{\prime}( & \left.\alpha_{1}\right) f_{\lambda, a}^{\prime}\left(\alpha_{2}\right) f_{\lambda, a}^{\prime}\left(\alpha_{3}\right) f_{\lambda, a}^{\prime}\left(\alpha_{4}\right) f_{\lambda, a}^{\prime}\left(\alpha_{5}\right) f_{\lambda, a}^{\prime}\left(\alpha_{6}\right) f_{\lambda, a}^{\prime}\left(\alpha_{7}\right) f_{\lambda, a}^{\prime}\left(\alpha_{8}\right) \mid \\
= & \mid 1.16505 \times(-2.04173) \times(-0.02740) \\
& \times(-2.26161) \times 1.36444 \times(-1.97047) \\
& \times(-0.31780) \times(-2.23880) \mid \approx 0.28197<1 .
\end{aligned}
$$

It provides that the periodic 8 -cycle of $f_{2.58,0.1}(x)$ is attracting

(iv) If $\lambda=2.592$, then the periodic points of 16 -cycle of $f_{\lambda, a}(x)$ are calculated as $\alpha_{1}=3.79505, \alpha_{2}=$ 12.35688, $\alpha_{3}=5.79026, \alpha_{4}=13.44454, \alpha_{5}=3.42588$, $\alpha_{6}=11.86701, \quad \alpha_{7}=6.77289, \quad \alpha_{8}=13.22379, \quad \alpha_{9}=$ $3.92537, \quad \alpha_{10}=12.50473, \quad \alpha_{11}=5.48341, \quad \alpha_{12}=$ $13.42254, \quad \alpha_{13}=3.47607, \quad \alpha_{14}=11.94007, \quad \alpha_{15}=$ 6.62972 , and $\alpha_{16}=13.28181$. It gives that

$$
\begin{aligned}
& \mid f_{\lambda, a}^{\prime}\left(\alpha_{1}\right) f_{\lambda, a}^{\prime}\left(\alpha_{2}\right) f_{\lambda, a}^{\prime}\left(\alpha_{3}\right) f_{\lambda, a}^{\prime}\left(\alpha_{4}\right) f_{\lambda, a}^{\prime}\left(\alpha_{5}\right) f_{\lambda, a}^{\prime}\left(\alpha_{6}\right) f_{\lambda, a}^{\prime} \\
& \quad \cdot\left(\alpha_{7}\right) f_{\lambda, a}^{\prime}\left(\alpha_{8}\right) f_{\lambda, a}^{\prime}\left(\alpha_{9}\right) f_{\lambda, a}^{\prime}\left(\alpha_{10}\right) \times f_{\lambda, a}^{\prime}\left(\alpha_{11}\right) f_{\lambda, a}^{\prime} \\
& \quad \cdot\left(\alpha_{12}\right) f_{\lambda, a}^{\prime}\left(\alpha_{13}\right) f_{\lambda, a}^{\prime}\left(\alpha_{14}\right) f_{\lambda, a}^{\prime}\left(\alpha_{15}\right) f_{\lambda, a}^{\prime}\left(\alpha_{16}\right) \mid \\
& =\mid 1.18286 \times(-2.05961) \times(-0.00301) \times(-2.28482) \\
& \quad \times 1.47652 \times(-1.95142) \times(-0.43436) \times(-2.24067) \\
& \quad \times 1.08659 \times(-2.09140) \times 0.14779 \times(-2.28046) \\
& \quad \times 1.43460 \times(-1.96785) \times(-0.37579) \times(-2.25234) \mid \\
& \approx 0 \\
& 0.08596<1 .
\end{aligned}
$$

Consequently, the periodic 16-cycle of $f_{2.592,0.1}(x)$ is attracting

For $a=0.2$, the periodic points of periods $2,4,8$, and 16 of $f_{\lambda, a}(x)$ are numerically simulated at the following values of parameter $\lambda=2.5,2.62,2.67,2.69$, respectively:

(i) If $\lambda=2.5$, then the periodic points of 2-cycle of $f_{\lambda, a}$ $(x)$ are obtained as $\beta_{1}=2.88749$ and $\beta_{2}=6.30188$. It follows that

$$
\begin{aligned}
\left|f_{\lambda, a}^{\prime}\left(\beta_{1}\right) f_{\lambda, a}^{\prime}\left(\beta_{2}\right)\right| & =0.21895 \times(-1.91984) \mid \\
& \approx 0.42035<1 .
\end{aligned}
$$


Therefore, the periodic 2-cycle of $f_{2.5,0.2}(x)$ is attracting

(ii) If $\lambda=2.62$, then the periodic points of 4-cycle of $f_{\lambda, a}(x)$ are found as $\beta_{1}=2.30475, \beta_{2}=6.20685, \beta_{3}$ $=2.90697$, and $\beta_{4}=6.49514$. It gives that

$$
\begin{aligned}
& \left|f_{\lambda, a}^{\prime}\left(\beta_{1}\right) f_{\lambda, a}^{\prime}\left(\beta_{2}\right) f_{\lambda, a}^{\prime}\left(\beta_{3}\right) f_{\lambda, a}^{\prime}\left(\beta_{4}\right)\right| \\
& \quad=|0.84298 \times(-2.02537) \times 0.14490 \times(-2.15148)| \\
& \quad \approx 0.53226<1 .
\end{aligned}
$$

Hence, the periodic 4-cycle of $f_{2.62,0.2}(x)$ is attracting

(iii) If $\lambda=2.67$, then the periodic points of 8 -cycle of $f_{\lambda, a}(x)$ are determined as $\beta_{1}=2.05248, \beta_{2}=$ 6.04153, $\beta_{3}=3.17847, \beta_{4}=6.57015, \beta_{5}=2.05248$, $\beta_{6}=6.04153, \beta_{7}=3.17847$, and $\beta_{8}=6.57015$. It follows that

$$
\begin{aligned}
& \mid f_{\lambda, a}^{\prime}\left(\beta_{1}\right) f_{\lambda, a}^{\prime}\left(\beta_{2}\right) f_{\lambda, a}^{\prime}\left(\beta_{3}\right) f_{\lambda, a}^{\prime}\left(\beta_{4}\right) f_{\lambda, a}^{\prime} \\
& \quad \cdot\left(\beta_{5}\right) f_{\lambda, a}^{\prime}\left(\beta_{6}\right) f_{\lambda, a}^{\prime}\left(\beta_{7}\right) f_{\lambda, a}^{\prime}\left(\beta_{8}\right) \mid \\
&=\mid 1.19456 \times(-2.00970) \times(-0.14577) \times(-2.24697) \\
& \quad \times 1.19456 \times(-2.00970) \times(-0.14577) \times(-2.24697) \mid \\
& \approx 0.61834<1 .
\end{aligned}
$$

It provides that the periodic 8 -cycle of $f_{2.67,0.2}(x)$ is attracting

(iv) If $\lambda=2.695$, then the periodic points of 16-cycle of $f_{\lambda, a}(x)$ are calculated as $\beta_{1}=1.91280, \beta_{2}=5.90522$, $\beta_{3}=3.42346, \quad \beta_{4}=6.53944, \quad \beta_{5}=2.07739, \quad \beta_{6}=$ 6.11643, $\beta_{7}=2.99582, \beta_{8}=6.61913, \beta_{9}=1.89548$, $\beta_{10}=5.88041, \beta_{11}=3.47228, \beta_{12}=6.51975, \beta_{13}=$ $2.12190, \beta_{14}=6.16627, \beta_{15}=2.89184$, and $\beta_{16}=$ 6.61159. It gives that

$$
\begin{aligned}
\mid f_{\lambda, a}^{\prime}\left(\beta_{1}\right) f_{\lambda, a}^{\prime}\left(\beta_{2}\right) f_{\lambda, a}^{\prime}\left(\beta_{3}\right) f_{\lambda, a}^{\prime}\left(\beta_{4}\right) f_{\lambda, a}^{\prime}\left(\beta_{5}\right) f_{\lambda, a}^{\prime}\left(\beta_{6}\right) f_{\lambda, a}^{\prime} \\
\quad \cdot\left(\beta_{7}\right) f_{\lambda, a}^{\prime}\left(\beta_{8}\right) f_{\lambda, a}^{\prime}\left(\beta_{9}\right) f_{\lambda, a}^{\prime}\left(\beta_{10}\right) \times f_{\lambda, a}^{\prime}\left(\beta_{11}\right) f_{\lambda, a}^{\prime} \\
\quad \cdot\left(\beta_{12}\right) f_{\lambda, a}^{\prime}\left(\beta_{13}\right) f_{\lambda, a}^{\prime}\left(\beta_{14}\right) f_{\lambda, a}^{\prime}\left(\beta_{15}\right) f_{\lambda, a}^{\prime}\left(\beta_{16}\right) \mid \\
=\mid 1.41733 \times(-1.97410) \times(-0.38207) \times(-2.26546) \\
\quad \times 1.15346 \times(-2.07466) \times 0.01923 \times(-2.29994) \\
\quad \times 1.44663 \times(-1.96204) \times(-0.42434) \times(-2.25687) \\
\quad \times 1.08622 \times(-2.09785) \times 0.12644 \times-2.29670) \mid \\
\approx 0.46100<1 .
\end{aligned}
$$

Consequently, the periodic 16-cycle of $f_{2.695,0.2}(x)$ is attracting

For $a=0.3$, the periodic points of periods $2,4,8$, and 16 of $f_{\lambda, a}(x)$ are numerically computed at the following values of parameter $\lambda=2.6,2.71,2.79,2.8$, respectively: (i) If $\lambda=2.6$, then the periodic points of 2-cycle of $f_{\lambda, a}$ $(x)$ are obtained as $\gamma_{1}=2.00940$ and $\gamma_{2}=4.14754$. It follows that

$$
\left|f_{\lambda, a}^{\prime}\left(\gamma_{1}\right) f_{\lambda, a}^{\prime}\left(\gamma_{2}\right)\right|=0.21362 \times(-1.92711) \mid \approx 0.41166<1 .
$$

Therefore, the periodic 2-cycle of $f_{2.6,0.3}(x)$ is attracting

(ii) If $\lambda=2.71$, then the periodic points of 4-cycle of $f_{\lambda, a}(x)$ are found as $\gamma_{1}=1.93317, \gamma_{2}=4.24257, \gamma_{3}$ $=1.71065$, and $\gamma_{4}=4.13613$. It gives that

$$
\begin{aligned}
& \left|f_{\lambda, a}^{\prime}\left(\gamma_{1}\right) f_{\lambda, a}^{\prime}\left(\gamma_{2}\right) f_{\lambda, a}^{\prime}\left(\gamma_{3}\right) f_{\lambda, a}^{\prime}\left(\gamma_{4}\right)\right| \\
& \quad=|0.28372 \times(-2.12793) \times 0.68242 \times(-2.05301)| \\
& \quad \approx 0.84584<1 .
\end{aligned}
$$

Hence, the periodic 4-cycle of $f_{2.71,0.3}(x)$ is attracting

(iii) If $\lambda=2.79$, then the periodic points of 8 -cycle of $f_{\lambda, a}(x)$ are determined as $\gamma_{1}=1.48950, \quad \gamma_{2}=$ $4.03152, \gamma_{3}=2.08267, \gamma_{4}=4.34522, \gamma_{5}=1.39642$, $\gamma_{6}=3.91613, \gamma_{7}=2.31675$, and $\gamma_{8}=4.30450$. It follows that

$$
\begin{aligned}
&\left|f_{\lambda, a}^{\prime}\left(\gamma_{1}\right) f_{\lambda, a}^{\prime}\left(\gamma_{2}\right) f_{\lambda, a}^{\prime}\left(\gamma_{3}\right) f_{\lambda, a}^{\prime}\left(\gamma_{4}\right) f_{\lambda, a}^{\prime}\left(\gamma_{5}\right) f_{\lambda, a}^{\prime}\left(\gamma_{6}\right) f_{\lambda, a}^{\prime}\left(\gamma_{7}\right) f_{\lambda, a}^{\prime}\left(\gamma_{8}\right)\right| \\
&=\mid 1.12881 \times(-2.07253) \times 0.00235 \times(-2.29950) \\
& \quad \times 1.35359 \times(-1.98420) \times(-0.34334) \times(-2.27105) \mid \\
& \approx 0.02653<1 .
\end{aligned}
$$

It provides that the periodic 8 -cycle of $f_{2.79,0.3}(x)$ is attracting

(iv) If $\lambda=2.8$, then the periodic points of 16-cycle of $f_{\lambda, a}(x)$ are calculated as $\gamma_{1}=1.51681, \gamma_{2}=4.07343$, $\gamma_{3}=1.98697, \quad \gamma_{4}=4.34796, \quad \gamma_{5}=1.37857, \quad \gamma_{6}=$ $3.90374, \gamma_{7}=2.33510, \gamma_{8}=4.30627, \gamma_{9}=1.47443$, $\gamma_{10}=4.02627, \quad \gamma_{11}=2.08592, \quad \gamma_{12}=4.35500, \gamma_{13}=$ $1.36226, \gamma_{14}=3.88060, \gamma_{15}=2.38085$, and $\gamma_{16}=$ 4.28767. It gives that

$$
\begin{aligned}
& \mid f_{\lambda, a}^{\prime}\left(\gamma_{1}\right) f_{\lambda, a}^{\prime}\left(\gamma_{2}\right) f_{\lambda, a}^{\prime}\left(\gamma_{3}\right) f_{\lambda, a}^{\prime}\left(\gamma_{4}\right) f_{\lambda, a}^{\prime}\left(\gamma_{5}\right) f_{\lambda, a}^{\prime} \\
& \quad \cdot\left(\gamma_{6}\right) f_{\lambda, a}^{\prime}\left(\gamma_{7}\right) f_{\lambda, a}^{\prime}\left(\gamma_{8}\right) f_{\lambda, a}^{\prime}\left(\gamma_{9}\right) f_{\lambda, a}^{\prime}\left(\gamma_{10}\right) \\
& \quad \times f_{\lambda, a}^{\prime}\left(\gamma_{11}\right) f_{\lambda, a}^{\prime}\left(\gamma_{12}\right) f_{\lambda, a}^{\prime}\left(\gamma_{13}\right) f_{\lambda, a}^{\prime}\left(\gamma_{14}\right) f_{\lambda, a}^{\prime}\left(\gamma_{15}\right) f_{\lambda, a}^{\prime}\left(\gamma_{16}\right) \mid \\
& =\mid 1.06390 \times(-2.11594) \times 0.15189 \times(-2.31406) \\
& \quad \times 1.39757 \times(-1.98613) \times(-0.37518) \times(-2.28486) \\
& \quad \times 1.16219 \times(-2.08046) \times(-0.00806) \times(-2.31897) \\
& \quad \times 1.43959 \times(-1.96795) \times(-0.43774) \times(-2.27173) \mid \\
& \approx \\
& \quad 0.23985<1 .
\end{aligned}
$$


Consequently, the periodic 16-cycle of $f_{2.8,0.3}(x)$ is attracting

For $a=0.9$, the periodic points of periods $2,4,8$, and 16 of $f_{\lambda, a}(x)$ are numerically computed at the following values of parameter $\lambda=3,3.35,3.4,3.412$, respectively:

(i) If $\lambda=3$, then the periodic points of 2-cycle of $f_{\lambda, a}(x)$ are obtained as $\rho_{1}=0.92614$ and $\rho_{2}=1.24997$. It follows that

$\left|f_{\lambda, a}^{\prime}\left(\rho_{1}\right) f_{\lambda, a}^{\prime}\left(\rho_{2}\right)\right|=|(-0.37396) \times(-1.55327)| \approx 0.58086<1$.

Thus, the periodic 2-cycle of $f_{3,0.9}(x)$ is attracting

(ii) If $\lambda=3.35$, then the periodic points of 4 -cycle of $f_{\lambda, a}(x)$ are found as $\rho_{1}=0.79227, \rho_{2}=1.35171$, $\rho_{3}=0.66014$, and $\rho_{4}=1.29091$. It gives that

$$
\begin{aligned}
& \left|f_{\lambda, a}^{\prime}\left(\rho_{1}\right) f_{\lambda, a}^{\prime}\left(\rho_{2}\right) f_{\lambda, a}^{\prime}\left(\rho_{3}\right) f_{\lambda, a}^{\prime}\left(\rho_{4}\right)\right| \\
& \quad=|0.04519 \times(-2.26683) \times 0.90905 \times(-2.07780)| \\
& \quad \approx 0.19347<1 .
\end{aligned}
$$

Hence, the periodic 4-cycle of $f_{3.35,0.9}(x)$ is attracting

(iii) If $\lambda=3.4$, then the periodic points of 8 -cycle of $f_{\lambda, a}(x)$ are determined as $\rho_{1}=0.61675, \rho_{2}=$ $1.26247, \rho_{3}=0.84200, \rho_{4}=1.35862, \rho_{5}=0.63074$, $\rho_{6}=1.27879, \rho_{7}=0.80822$, and $\rho_{8}=1.36456$. It follows that

$$
\begin{aligned}
&\left|f_{\lambda, a}^{\prime}\left(\rho_{1}\right) f_{\lambda, a}^{\prime}\left(\rho_{2}\right) f_{\lambda, a}^{\prime}\left(\rho_{3}\right) f_{\lambda, a}^{\prime}\left(\rho_{4}\right) f_{\lambda, a}^{\prime}\left(\rho_{5}\right) f_{\lambda, a}^{\prime}\left(\rho_{6}\right) f_{\lambda, a}^{\prime}\left(\rho_{7}\right) f_{\lambda, a}^{\prime}\left(\rho_{8}\right)\right| \\
&=\mid 1.22284 \times(-2.04211) \times(-0.26942) \times(-2.34773) \\
& \quad \times 1.11057 \times(-2.09591) \times(-0.08058) \times(-2.36576) \mid \\
& \approx 0.70089<1 .
\end{aligned}
$$

It provides that the periodic 8-cycle of $f_{3.4,0.9}(x)$ is attracting

(iv) If $\lambda=3.412$, then the periodic points of 16-cycle of $f_{\lambda, a}(x)$ are calculated as $\rho_{1}=0.60493, \rho_{2}=1.25186$, $\rho_{3}=0.86169, \quad \rho_{4}=1.35490, \quad \rho_{5}=0.63623, \quad \rho_{6}=$ $1.28903, \rho_{7}=0.78430, \rho_{8}=1.36810, \rho_{9}=0.60493$, $\rho_{10}=1.25186, \rho_{11}=0.86169, \rho_{12}=1.35490, \rho_{13}=$ $0.63623, \rho_{14}=1.28903, \rho_{15}=0.78430$, and $\rho_{16}=$ 1.36810. It gives that

$$
\begin{aligned}
& \mid f_{\lambda, a}^{\prime}\left(\rho_{1}\right) f_{\lambda, a}^{\prime}\left(\rho_{2}\right) f_{\lambda, a}^{\prime}\left(\rho_{3}\right) f_{\lambda, a}^{\prime}\left(\rho_{4}\right) f_{\lambda, a}^{\prime}\left(\rho_{5}\right) f_{\lambda, a}^{\prime}\left(\rho_{6}\right) f_{\lambda, a}^{\prime} \\
& \quad \cdot\left(\rho_{7}\right) f_{\lambda, a}^{\prime}\left(\rho_{8}\right) f_{\lambda, a}^{\prime}\left(\rho_{9}\right) f_{\lambda, a}^{\prime}\left(\rho_{10}\right) \times f_{\lambda, a}^{\prime}\left(\rho_{11}\right) f_{\lambda, a}^{\prime} \\
& \quad \cdot\left(\rho_{12}\right) f_{\lambda, a}^{\prime}\left(\rho_{13}\right) f_{\lambda, a}^{\prime}\left(\rho_{14}\right) f_{\lambda, a}^{\prime}\left(\rho_{15}\right) f_{\lambda, a}^{\prime}\left(\rho_{16}\right) \mid \\
&=\mid 1.31559 \times(-2.02013) \times(-0.38409) \times(-2.35076) \\
& \quad \times 1.06211 \times(-2.14301) \times(0.05149) \times(-2.39098) \\
& \quad \times 1.31559 \times(-2.02013) \times(-0.38409) \times(-2.35076) \\
& \quad \times 1.06211 \times(-2.14301) \times 0.05149 \times(-2.39098) \mid \\
& \approx 0.45220<1 .
\end{aligned}
$$

Consequently, the periodic 16-cycle of $f_{3.412,0.9}(x)$ is attracting

For $a=1$, the periodic points of periods $2,4,8$, and 16 of $f_{\lambda, a}(x)$ are numerically simulated at the following values of parameter $\lambda=3.2,3.43,3.51,3.52$, respectively:

(i) If $\lambda=3.2$, then the periodic points of 2-cycle of $f_{\lambda, a}$ $(x)$ are obtained as $\sigma_{1}=0.77084$ and $\sigma_{2}=1.15258$. It follows that

$$
\begin{aligned}
\left|f_{\lambda, a}^{\prime}\left(\sigma_{1}\right) f_{\lambda, a}^{\prime}\left(\sigma_{2}\right)\right| & =|(-0.06982) \times(-1.78680)| \\
& \approx 0.12475<1 .
\end{aligned}
$$

Hence, the periodic 2-cycle of $f_{3.2,1}(x)$ is attracting

(ii) If $\lambda=3.43$, then the periodic points of 4-cycle of $f_{\lambda, a}(x)$ are found as $\sigma_{1}=0.70235, \sigma_{2}=1.20020, \sigma_{3}$ $=0.63143$, and $\sigma_{4}=1.16743$. It gives that

$$
\begin{aligned}
& \left|f_{\lambda, a}^{\prime}\left(\sigma_{1}\right) f_{\lambda, a}^{\prime}\left(\sigma_{2}\right) f_{\lambda, a}^{\prime}\left(\sigma_{3}\right) f_{\lambda, a}^{\prime}\left(\sigma_{4}\right)\right| \\
& \quad=|0.20572 \times(-2.22275) \times 0.73067 \times(-2.10441)| \\
& \quad \approx 0.70312<1 .
\end{aligned}
$$

It provides that the periodic 4 -cycle of $f_{3.43,1}(x)$ is attracting

(iii) If $\lambda=3.51$, then the periodic points of 8 -cycle of $f_{\lambda, a}(x)$ are determined as $\sigma_{1}=0.77980, \sigma_{2}=$ $1.21185, \sigma_{3}=0.58668, \sigma_{4}=1.15156, \sigma_{5}=0.72229$, $\sigma_{6}=1.22175, \sigma_{7}=0.56315$, and $\sigma_{8}=1.12396$. It follows that

$$
\begin{aligned}
& \mid f_{\lambda, a}^{\prime}\left(\sigma_{1}\right) f_{\lambda, a}^{\prime}\left(\sigma_{2}\right) f_{\lambda, a}^{\prime}\left(\sigma_{3}\right) f_{\lambda, a}^{\prime}\left(\sigma_{4}\right) f_{\lambda, a}^{\prime} \\
& \quad \cdot\left(\sigma_{5}\right) f_{\lambda, a}^{\prime}\left(\sigma_{6}\right) f_{\lambda, a}^{\prime}\left(\sigma_{7}\right) f_{\lambda, a}^{\prime}\left(\sigma_{8}\right) \mid \\
& =\mid(-0.35462) \times(-2.35925) \times 1.06627 \times(-2.13692) \\
& \quad \times 0.01636 \times(-2.39449) \times 1.28116 \times(-2.03046) \mid \\
& \approx 0.19425<1 .
\end{aligned}
$$

Therefore, the periodic 8-cycle of $f_{3.51,1}(x)$ is attracting 
(iv) If $\lambda=3.52$, then the periodic points of 16-cycle of $f_{\lambda, a}(x)$ are calculated as $\sigma_{1}=0.70033, \sigma_{2}=1.22223$, $\sigma_{3}=0.55955, \quad \sigma_{4}=1.12253, \quad \sigma_{5}=0.78139, \quad \sigma_{6}=$ $1.21320, \sigma_{7}=0.58114, \sigma_{8}=1.14866, \sigma_{9}=0.72687$, $\sigma_{10}=1.22407, \sigma_{11}=0.55511, \sigma_{12}=1.11661, \sigma_{13}=$ $0.79338, \sigma_{14}=1.20830, \sigma_{15}=0.59273$, and $\sigma_{16}=$ 1.16094. It gives that

$$
\begin{aligned}
& \mid f_{\lambda, a}^{\prime}\left(\sigma_{1}\right) f_{\lambda, a}^{\prime}\left(\sigma_{2}\right) f_{\lambda, a}^{\prime}\left(\sigma_{3}\right) f_{\lambda, a}^{\prime}\left(\sigma_{4}\right) f_{\lambda, a}^{\prime}\left(\sigma_{5}\right) f_{\lambda, a}^{\prime}\left(\sigma_{6}\right) f_{\lambda, a}^{\prime} \\
& \quad \cdot\left(\rho_{7}\right) f_{\lambda, a}^{\prime}\left(\sigma_{8}\right) f_{\lambda, a}^{\prime}\left(\sigma_{9}\right) f_{\lambda, a}^{\prime}\left(\sigma_{10}\right) \times f_{\lambda, a}^{\prime}\left(\sigma_{11}\right) f_{\lambda, a}^{\prime} \\
& \quad \cdot\left(\sigma_{12}\right) f_{\lambda, a}^{\prime}\left(\sigma_{13}\right) f_{\lambda, a}^{\prime}\left(\sigma_{14}\right) f_{\lambda, a}^{\prime}\left(\sigma_{15}\right) f_{\lambda, a}^{\prime}\left(\sigma_{16}\right) \mid \\
&=\mid 0.16171 \times(-2.40819) \times 1.31092 \times(-2.03603) \\
& \quad \times(-0.37194) \times(-2.37602) \times 1.11127 \times(-2.13729) \\
& \quad \times(-0.02132) \times(-2.41473) \times 1.35330 \times(-2.01269) \\
& \quad \times(-0.44486) \times(-2.35844) \times 1.00810 \times(-2.18393) \mid \\
& \approx 0.70651<1 .
\end{aligned}
$$

Consequently, the periodic 16-cycle of $f_{3.52,1}(x)$ is attracting

For $a=1.1$, the periodic points of periods $2,4,8$, and 16 of $f_{\lambda, a}(x)$ are numerically calculated at the following parameter $\lambda=3.3,3.55,3.61,3.62$, respectively:

(i) If $\lambda=3.3$, then the periodic 2-cycle points of $f_{\lambda, a}(x)$ are obtained as $\tau_{1}=0.70921$ and $\tau_{2}=1.04202$. It follows that

$$
\begin{aligned}
\left|f_{\lambda, a}^{\prime}\left(\tau_{1}\right) f_{\lambda, a}^{\prime}\left(\tau_{2}\right)\right| & =|(-0.07062) \times(-1.79068)| \\
& \approx 0.12645<1 .
\end{aligned}
$$

Therefore, the periodic 2-cycle of $f_{3.3,1.1}(x)$ is attracting

(ii) If $\lambda=3.55$, then the periodic points of 4 -cycle of $f_{\lambda, a}(x)$ are found as $\tau_{1}=0.66490, \tau_{2}=1.09044, \tau_{3}$ $=0.56821$, and $\tau_{4}=1.04622$. It gives that

$$
\begin{aligned}
& \left|f_{\lambda, a}^{\prime}\left(\tau_{1}\right) f_{\lambda, a}^{\prime}\left(\tau_{2}\right) f_{\lambda, a}^{\prime}\left(\tau_{3}\right) f_{\lambda, a}^{\prime}\left(\tau_{4}\right)\right| \\
& \quad=|0.06443 \times(-2.27867) \times 0.87824 \times(-2.09294)| \\
& \quad \approx 0.26987<1 .
\end{aligned}
$$

Hence, the periodic 4-cycle of $f_{3.55,1.1}(x)$ is attracting

(iii) If $\lambda=3.61$, then the periodic points of 8-cycle of $f_{\lambda, a}(x)$ are determined as $\tau_{1}=0.54556, \tau_{2}=$ $1.04060, \tau_{3}=0.66816, \tau_{4}=1.10296, \tau_{5}=0.52658$, $\tau_{6}=1.01854, \tau_{7}=0.71422$, and $\tau_{8}=1.09500$. It follows that

$$
\begin{aligned}
&\left|f_{\lambda, a}^{\prime}\left(\tau_{1}\right) f_{\lambda, a}^{\prime}\left(\tau_{2}\right) f_{\lambda, a}^{\prime}\left(\tau_{3}\right) f_{\lambda, a}^{\prime}\left(\tau_{4}\right) f_{\lambda, a}^{\prime}\left(\tau_{5}\right) f_{\lambda, a}^{\prime}\left(\tau_{6}\right) f_{\lambda, a}^{\prime}\left(\tau_{7}\right) f_{\lambda, a}^{\prime}\left(\tau_{8}\right)\right| \\
&=\mid 1.06636 \times(-2.13677) \times(-0.00176) \times(-2.40118) \\
& \quad \times 1.26021 \times(-2.03861) \times(-0.33893) \times(-2.36845) \mid \\
& \approx 0.01989<1 .
\end{aligned}
$$

It provides that the periodic 8 -cycle of $f_{3.61,1.1}(x)$ is attracting

(iv) If $\lambda=3.62$, then the periodic points of 16-cycle of $f_{\lambda, a}(x)$ are calculated as $\tau_{1}=0.52206, \tau_{2}=1.01563$, $\tau_{3}=0.71902, \quad \tau_{4}=1.09498, \quad \tau_{5}=0.54358, \quad \tau_{6}=$ $1.04126, \tau_{7}=0.66533, \tau_{8}=1.10501, \tau_{9}=0.51949$, $\tau_{10}=1.01225, \tau_{11}=0.72587, \tau_{12}=1.09221, \tau_{13}=$ $0.55014, \tau_{14}=1.04815, \tau_{15}=0.65042$, and $\tau_{16}=$ 1.10395. It gives that

$$
\begin{aligned}
& \mid f_{\lambda, a}^{\prime}\left(\tau_{1}\right) f_{\lambda, a}^{\prime}\left(\tau_{2}\right) f_{\lambda, a}^{\prime}\left(\tau_{3}\right) f_{\lambda, a}^{\prime}\left(\tau_{4}\right) f_{\lambda, a}^{\prime}\left(\tau_{5}\right) f_{\lambda, a}^{\prime}\left(\tau_{6}\right) f_{\lambda, a}^{\prime} \\
& \quad \cdot\left(\rho_{7}\right) f_{\lambda, a}^{\prime}\left(\tau_{8}\right) f_{\lambda, a}^{\prime}\left(\tau_{9}\right) f_{\lambda, a}^{\prime}\left(\tau_{10}\right) f_{\lambda, a}^{\prime}\left(\tau_{11}\right) \times f_{\lambda, a}^{\prime} \\
& \quad \cdot\left(\tau_{12}\right) f_{\lambda, a}^{\prime}\left(\tau_{13}\right) f_{\lambda, a}^{\prime}\left(\tau_{14}\right) f_{\lambda, a}^{\prime}\left(\tau_{15}\right) f_{\lambda, a}^{\prime}\left(\tau_{16}\right) \mid \\
&=\mid 1.30341 \times(-2.03653) \times(-0.38015) \times(-2.38022) \\
& \quad \times 1.08133 \times(-2.15103) \times 0.01303 \times(-2.42152) \\
& \quad \times 1.33067 \times(-2.02120) \times(-0.42757) \times(-2.36875) \\
& \quad \times 1.01591 \times(-2.18120) \times 0.12957 \times(-2.41718) \mid \\
& \approx 0.33319<1 .
\end{aligned}
$$

Consequently, the periodic 16-cycle of $f_{3.62,1.1}(x)$ is attracting

\section{Numerical Simulation of Real Cycles of Period 3 with Their Nature}

The real cycle of period 3 corresponding to $\lambda=$ $2.782,2.892,3,3.635,3.735,3.84$ with their nature is determined for $a=0.1,0.2,0.3,0.9,1,1.1$, respectively, as follows.

(i) For $a=0.1$ and $\lambda=2.782$, the periodic points of 3cycle of $f_{\lambda, a}(x)$ are found as $\alpha_{1}=-14.05325, \alpha_{2}=$ 1.09033 , and $\alpha_{3}=5.59632$. It follows that

$$
\begin{aligned}
& \left|f_{\lambda, a}^{\prime}\left(\alpha_{1}\right) f_{\lambda, a}^{\prime}\left(\alpha_{2}\right) f_{\lambda, a}^{\prime}\left(\alpha_{3}\right)\right| \\
& \quad=|(-2.65747) \times 5.30037 \times 0.01157| \\
& \quad \approx 0.16302<1 .
\end{aligned}
$$

Therefore, the periodic 3-cycle of $f_{2.782,0.1}(x)$ is attracting

(ii) For $a=0.2$ and $\lambda=2.892$, the periodic points of 3cycle of $f_{\lambda, a}(x)$ are calculated as $\beta_{1}=2.88261, \beta_{2}=$ 6.92313 , and $\beta_{3}=0.73290$. It follows that 


$$
\begin{aligned}
& \left|f_{\lambda, a}^{\prime}\left(\beta_{1}\right) f_{\lambda, a}^{\prime}\left(\beta_{2}\right) f_{\lambda, a}^{\prime}\left(\beta_{3}\right)\right| \\
& \quad=|0.04765 \times(-2.68870) \times 5.02564| \\
& \quad \approx 0.64388<1 .
\end{aligned}
$$

Consequently, the periodic 3-cycle of $f_{2.892,0.2}(x)$ is attracting

(iii) For $a=0.3$ and $\lambda=3$, the periodic points of 3 -cycle of $f_{\lambda, a}(x)$ are determined as $\gamma_{1}=0.60380, \gamma_{2}=$ 1.99006, and $\gamma_{3}=4.55372$. It gives that

$$
\begin{aligned}
& \left|f_{\lambda, a}^{\prime}\left(\gamma_{1}\right) f_{\lambda, a}^{\prime}\left(\gamma_{2}\right) f_{\lambda, a}^{\prime}\left(\gamma_{3}\right)\right| \\
& \quad=|4.78163 \times 0.04991 \times(-2.71631)| \\
& \quad \approx 0.64832<1 .
\end{aligned}
$$

Therefore, the periodic 3-cycle of $f_{3,0.3}(x)$ is attracting

(iv) For $a=0.9$ and $\lambda=3.635$, the periodic points of 3 cycle of $f_{\lambda, a}(x)$ are given as $\rho_{1}=0.75942, \rho_{2}=$ 1.42939 , and $\rho_{3}=0.37250$. It follows that

$$
\begin{aligned}
\left|f_{\lambda, a}^{\prime}\left(\rho_{1}\right) f_{\lambda, a}^{\prime}\left(\rho_{2}\right) f_{\lambda, a}^{\prime}\left(\rho_{3}\right)\right| & =|0.06512 \times(-2.85102) \times 4.02216| \\
& \approx 0.74674<1 .
\end{aligned}
$$

Thus, the periodic 3-cycle of $f_{3.635,0.9}(x)$ is attracting

(v) For $a=1$ and $\lambda=3.735$, the periodic points of 3 cycle of $f_{\lambda, a}(x)$ are computed as $\sigma_{1}=0.70636, \sigma_{2}=$ 1.27587 , and $\sigma_{3}=0.35853$. It gives that

$$
\begin{aligned}
& \left|f_{\lambda, a}^{\prime}\left(\sigma_{1}\right) f_{\lambda, a}^{\prime}\left(\sigma_{2}\right) f_{\lambda, a}^{\prime}\left(\sigma_{3}\right)\right| \\
& \quad=|(-0.02088) \times(-2.86116) \times 3.88537| \\
& \quad \approx 0.23208<1 .
\end{aligned}
$$

It provides that the periodic 3 -cycle of $f_{3.735,1}(x)$ is attracting

(vi) For $a=1.1$ and $\lambda=3.84$, the periodic points of 3 cycle of $f_{\lambda, a}(x)$ are found as $\tau_{1}=0.34162, \tau_{2}=$ 0.64682 , and $\tau_{3}=1.15187$. It gives that

$$
\begin{aligned}
\left|f_{\lambda, a}^{\prime}\left(\tau_{1}\right) f_{\lambda, a}^{\prime}\left(\tau_{2}\right) f_{\lambda, a}^{\prime}\left(\tau_{3}\right)\right| & =|3.84560 \times 0.01311 \times(-2.88075)| \\
& \approx 0.14520<1 .
\end{aligned}
$$

Therefore, the periodic 3-cycle of $f_{3.84,1.1}(x)$ is attracting

The presence of periodic points of periods more than or equal to 2 can be visualized by bifurcation diagrams, and presence of chaotic behaviour in our dynamical systems can be quantified by Lyapunov exponents which are given in the following section.

\section{Chaos by Bifurcation Diagrams and Lyapunov Exponents}

In this section, we observe chaos in the real dynamics of $f_{\lambda, a} \in \mathscr{F}$ by portraying bifurcation diagrams and by computing Lyapunov exponents.

5.1. Period-Doubling and Period 3 Window in Bifurcation Diagrams. The purpose of this section is to visualize graphically the dynamical behaviour of $f_{\lambda, a} \in \mathscr{F}$ by using graphs of bifurcation diagrams. By drawing bifurcation diagrams, it is easy to see the periodicity of orbits graphically. It is familiar that the occurrence of period-doubling in the real dynamics of functions is route to chaos, and moreover, the periodic point of period three implies chaos [50]. It is observed from Section 2.1 that the stability of fixed points of $f_{\lambda, a}(x)$ varies whenever parameter $\lambda$ crosses a certain value. The appearance of periodic points of period two or greater in the real dynamics is exhibited through bifurcation diagrams (Figures 2(a)-2(f)) for $a=0.1,0.2,0.3,0.9,1,1.1$ with $2 \leq \lambda \leq 2.8,2 \leq \lambda \leq 2.9,2 \leq \lambda \leq 3.05,2.7 \leq \lambda \leq 3.7,2.8$ $\leq \lambda \leq 3.8$, and $2.9 \leq \lambda \leq 3.9$, respectively. In these bifurcation diagrams, the period-doubling occurs which presents a route to chaos in the dynamics of $f_{\lambda, a} \in \mathscr{F}$.

When a varies from 0.1 to 1.1 , it is clear from Figures 2(a)-2(f) that the period-doubling in bifurcation diagrams happens for little larger values of parameter $\lambda$. In these figures, white regions indicate the presence of nonchaotic windows in the bifurcation diagrams. This chaotic behaviour is observed in the bifurcation diagrams for very short interval of parameter $\lambda$. If $a$ varies from 0.1 to 1.1 , then the dark region in bifurcation diagrams shifted to little larger value of $\lambda$.

From the above discussion and numerical calculation, it is evidenced that saddle-node bifurcation happens in the dynamics of $f_{\lambda, a}(x)$ which is also demonstrated in bifurcation diagrams; see Figures 2(a)-2(f) and Figures 3(a)-3(f).

After zooming in the above bifurcation diagrams, a periodic-three window is clearly visible in these diagrams of $f_{\lambda, a}(x)$ for $a=0.1,0.2,0.3,0.9,1,1.1$ corresponding to $\lambda=$ 2.782,2.892, 3,3.635,3.735,3.84, respectively (Figures 3(a)$3(\mathrm{f})$ ) which interprets chaotic behaviour in the dynamics. For numerical computation of the bifurcation points, we solve the equations associated to fixed points and neutral fixed point conditions as $f_{\lambda, a}^{3}(x)=x$ and $\left|\left(f_{\lambda, a}^{3}\right)^{\prime}(x)\right|=1$.

We can observe ahead of the periodic-3 window, new higher periodic cycles come into existence. Beyond that dynamics exhibits extremely more complicated behaviour. It demonstrates that the real dynamics of $f_{\lambda, a}(x)$ changes suddenly under small changes in the parameters.

Next, the Lyapunov exponents are plotted corresponding to values of $\lambda$ and $a$ for which period-doubling happens in the above bifurcation diagrams. 


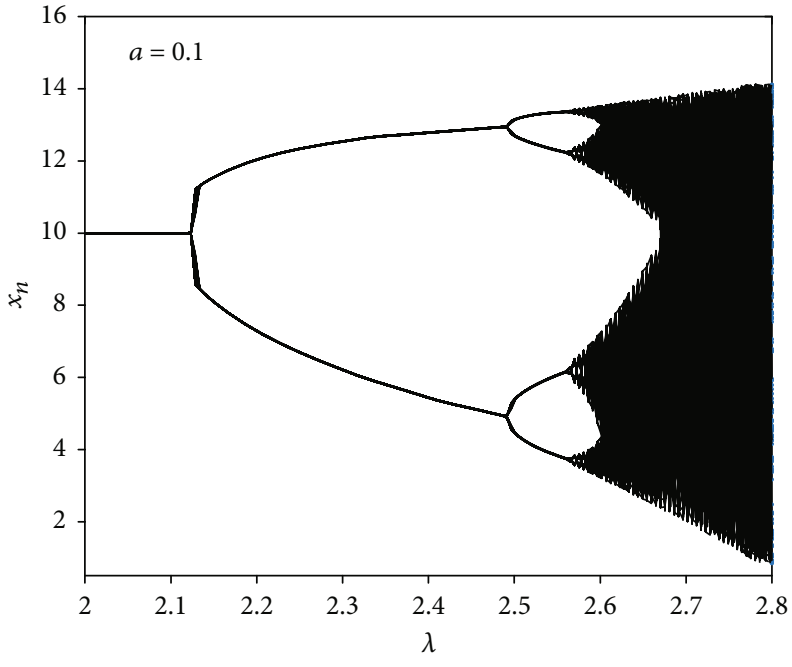

(a)

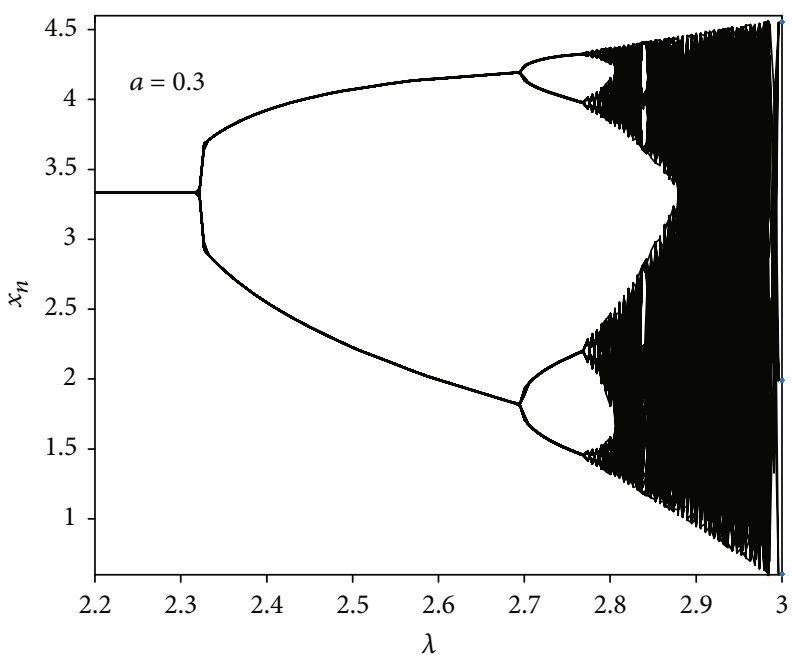

(c)

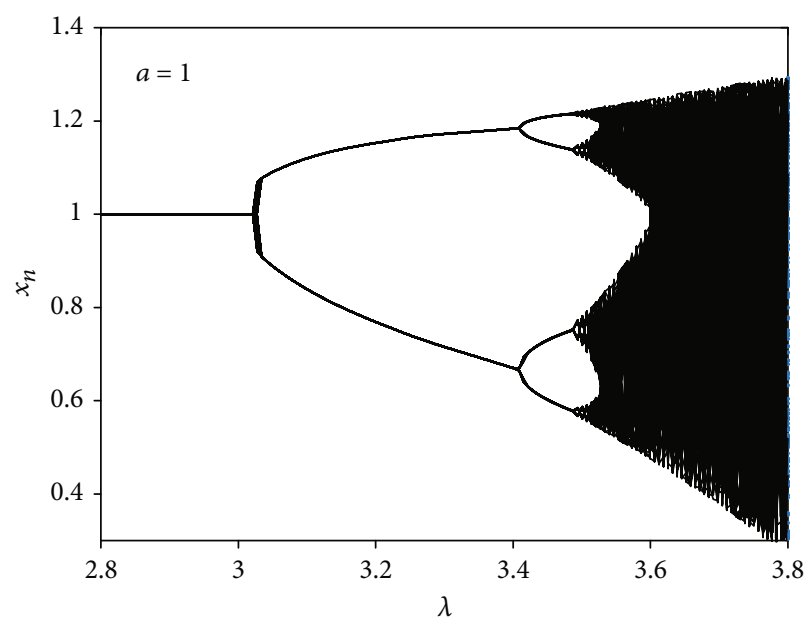

(e)

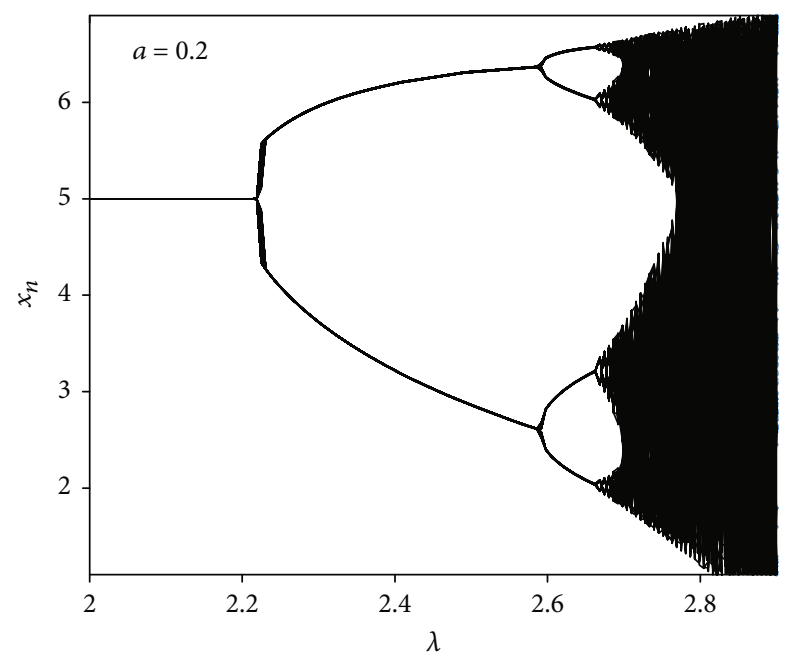

(b)

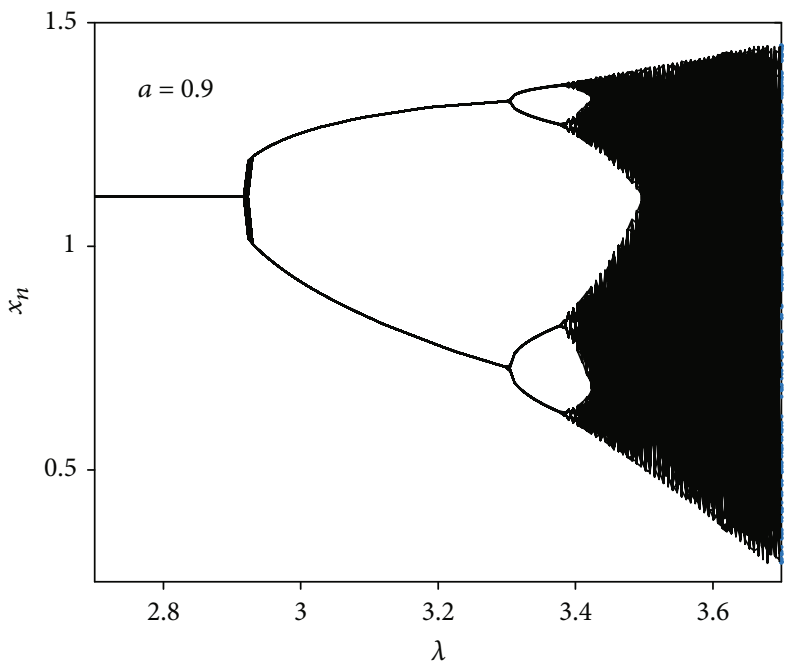

(d)

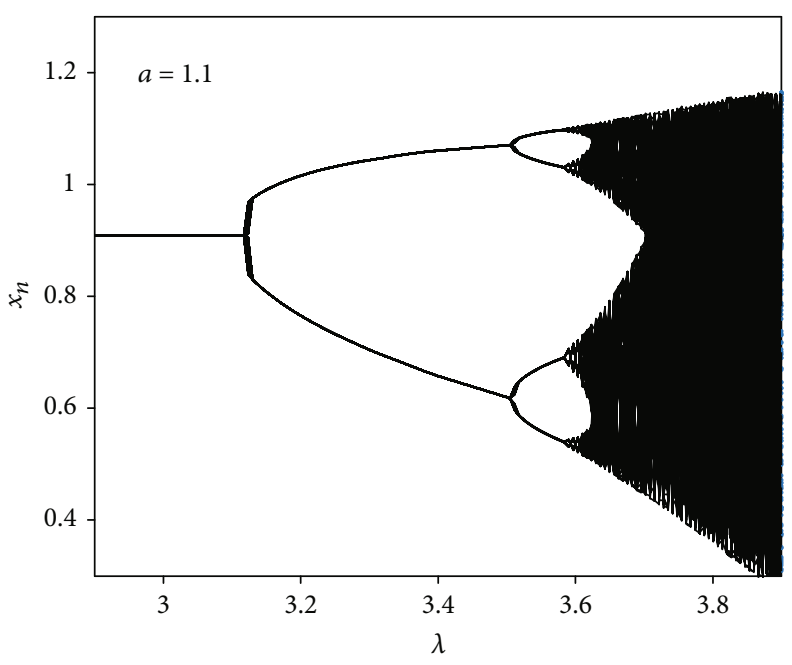

(f)

FIgURE 2: Bifurcation diagrams for $a=0.1, a=0.2, a=0.3, a=0.9, a=1$, and $a=1.1$. 


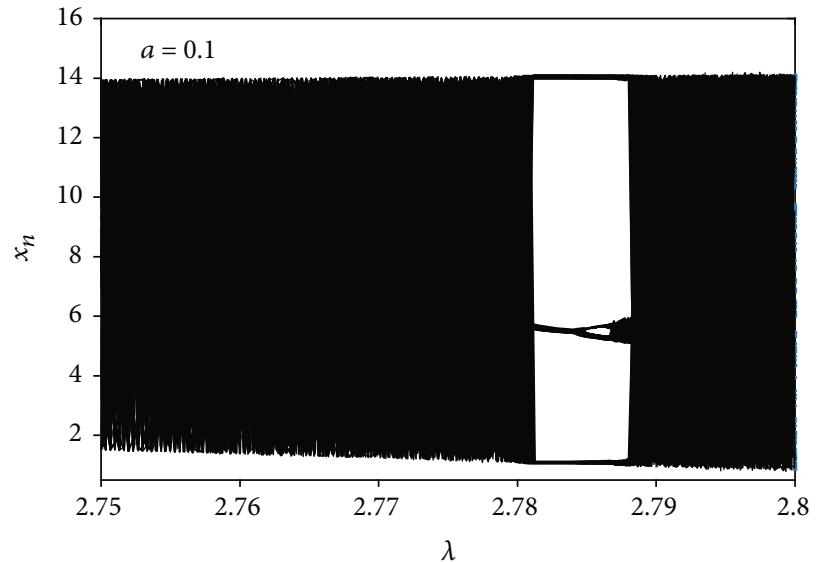

(a)

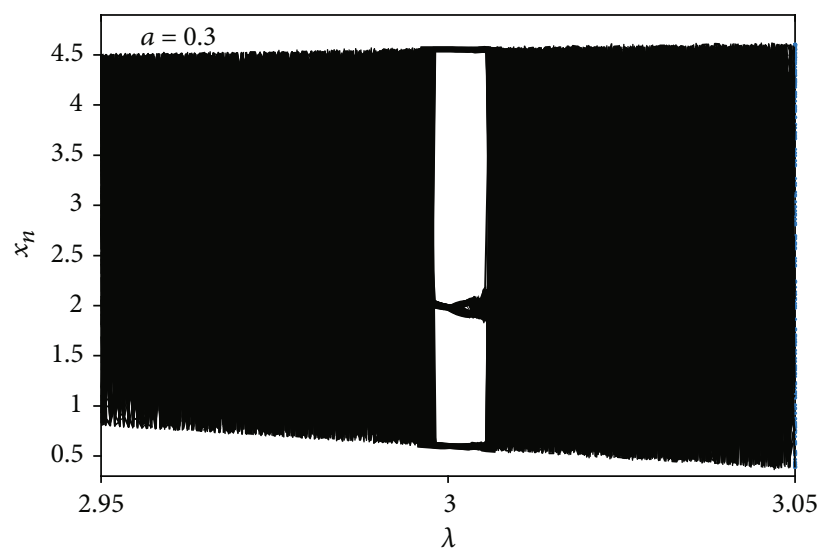

(c)

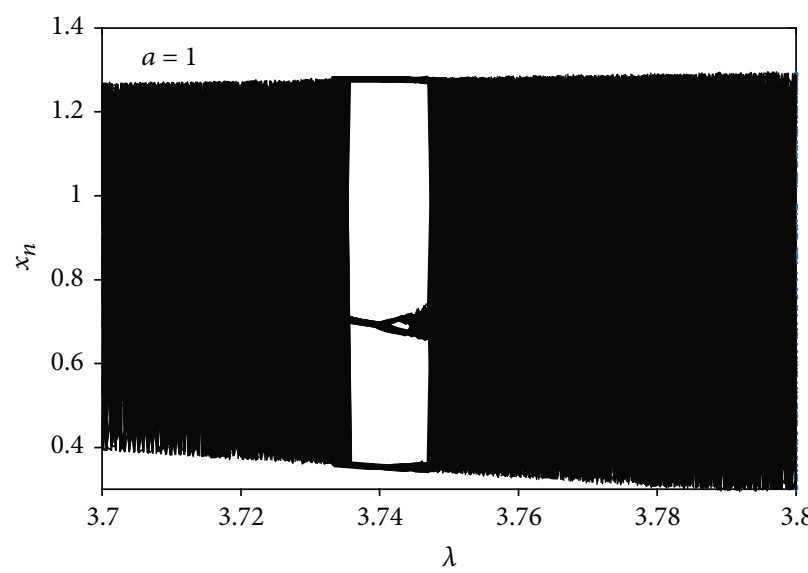

(e)

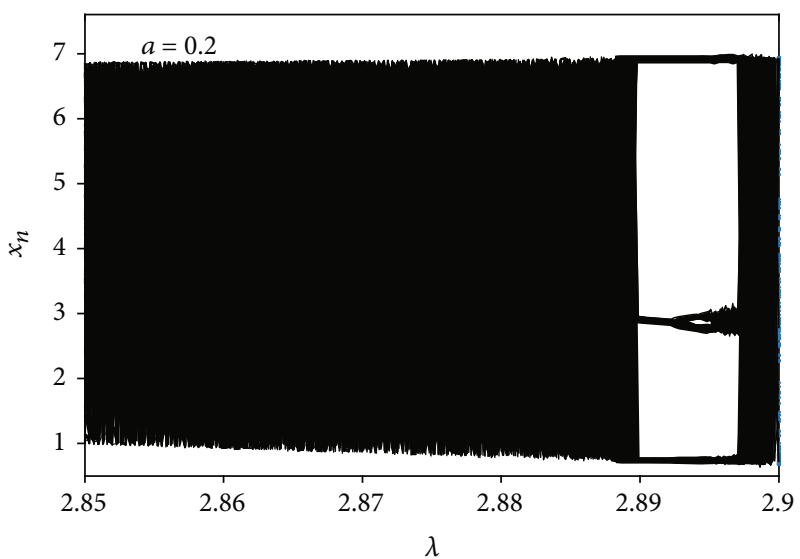

(b)

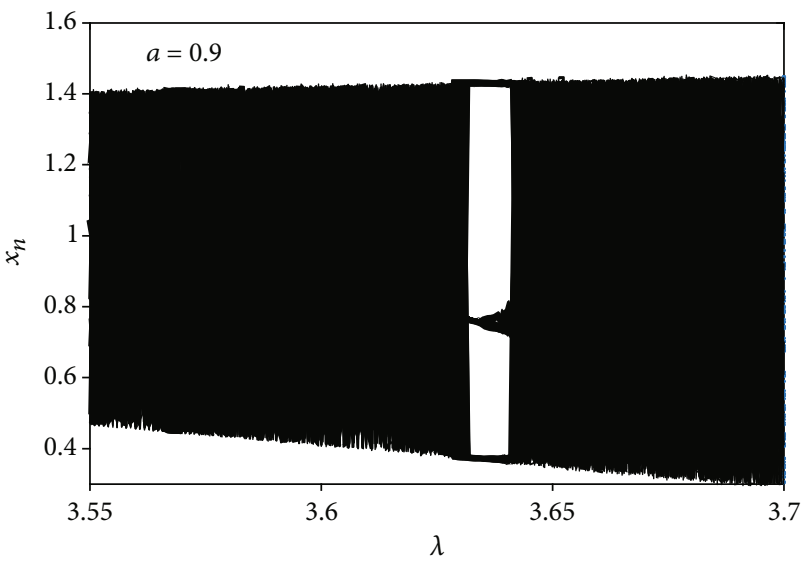

(d)

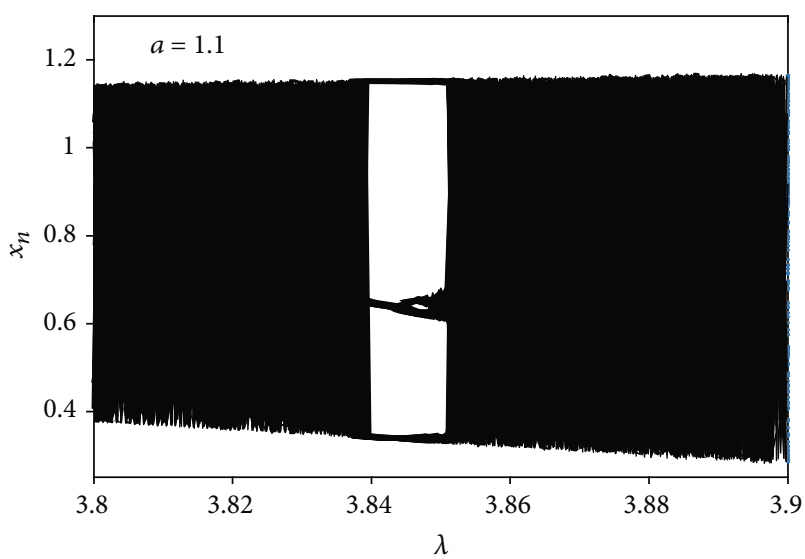

(f)

FIgURE 3: Bifurcation diagrams for period-3 window.

5.2. Lyapunov Exponents and Chaotic Behaviour. We are familiar that the chaotic behaviour happens in the dynamics of functions if the Lyapunov exponents are positive [50]. To compute the Lyapunov exponent of $f_{\lambda, a} \in \mathscr{F}$, formula (1) is used:

$$
L=\lim _{j \rightarrow \infty} \frac{1}{j} \sum_{k=0}^{j-1} \ln \left|1+\frac{1}{x_{k}}-\lambda\left(1+\ln a x_{k}\right)\right| .
$$

We pick $j=5000$ and $x_{0}=0.4$ in our computation. In Figure 4 , the calculated values of Lyapunov exponents are plotted for $a=0.1,0.2,0.3,0.9,1,1.1$ with $2.6 \leq \lambda \leq 2.8,2.6 \leq$ $\lambda \leq 2.9,2.6 \leq \lambda \leq 3.05,2.6 \leq \lambda \leq 3.7,2.6 \leq \lambda \leq 3.8$, and $2.6 \leq$ $\lambda \leq 3.9$, respectively. For certain intervals of parameter $\lambda$, it is seen that the Lyapunov exponents are positive. Therefore, this shows that chaotic behaviour presences in the real dynamics of $f_{\lambda, a} \in \mathscr{F}$. 


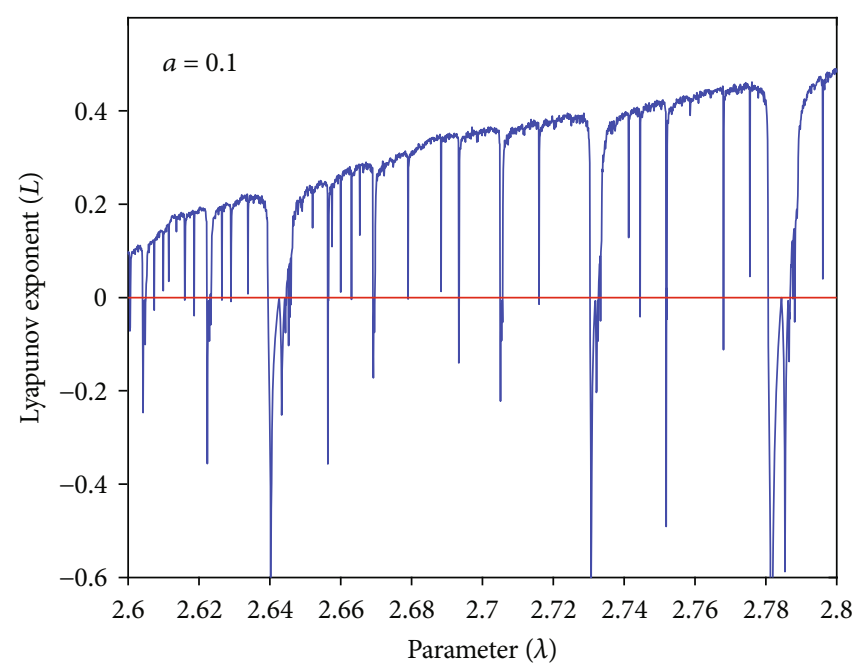

(a)

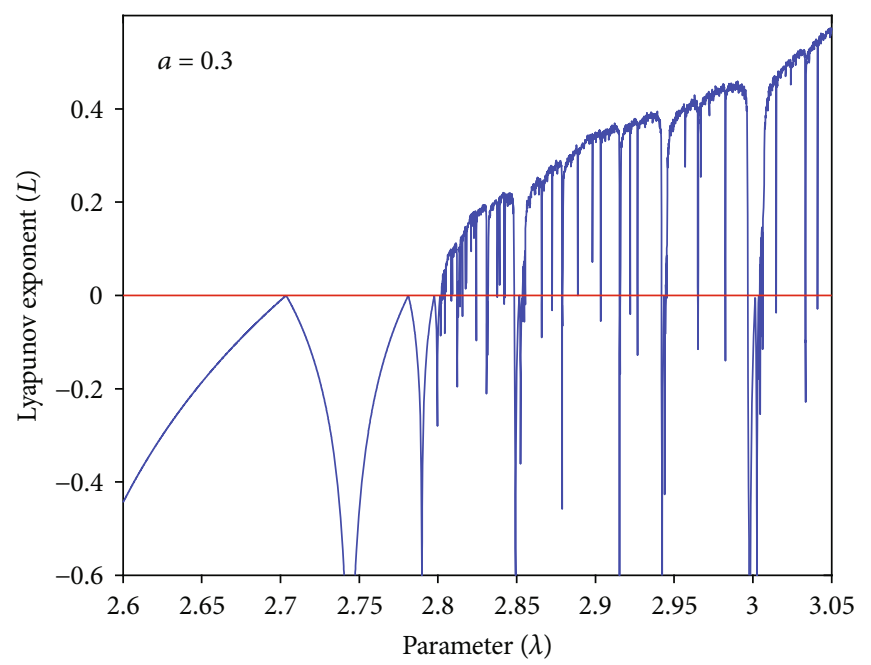

(c)

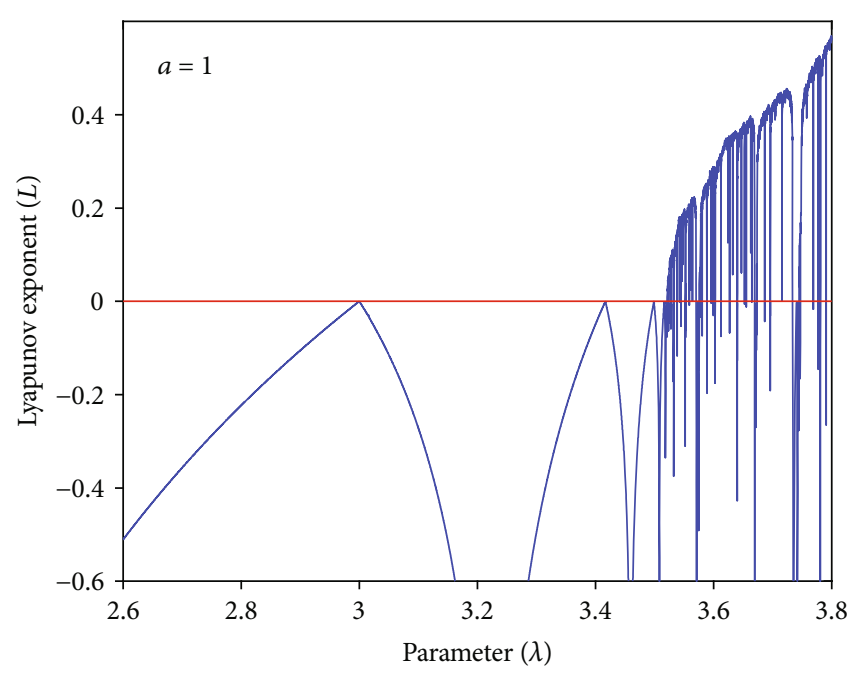

(e)

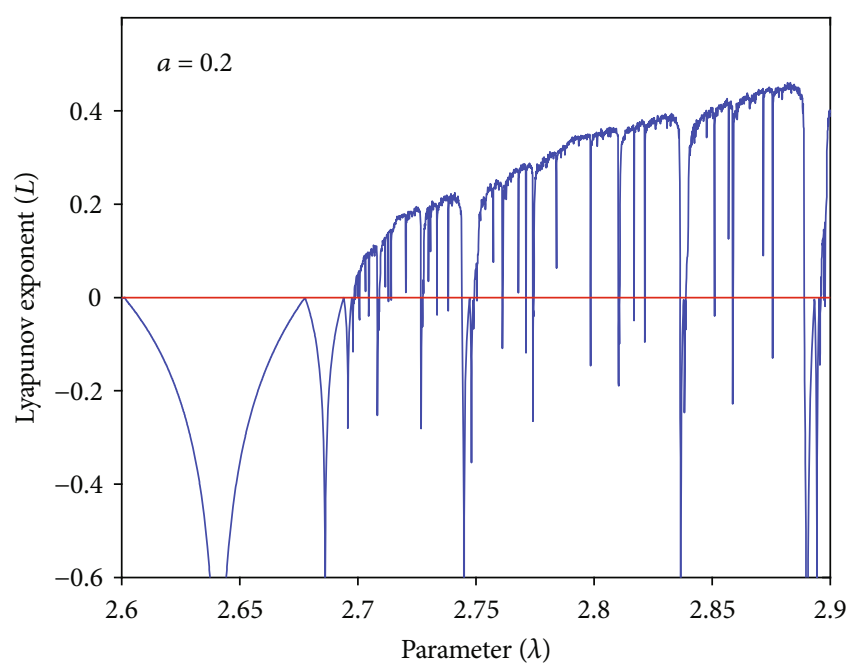

(b)

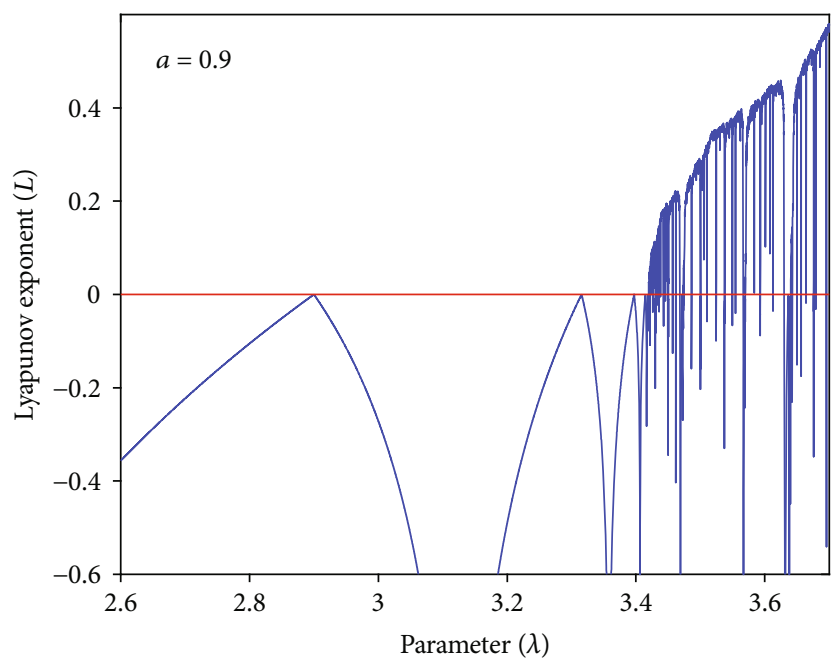

(d)

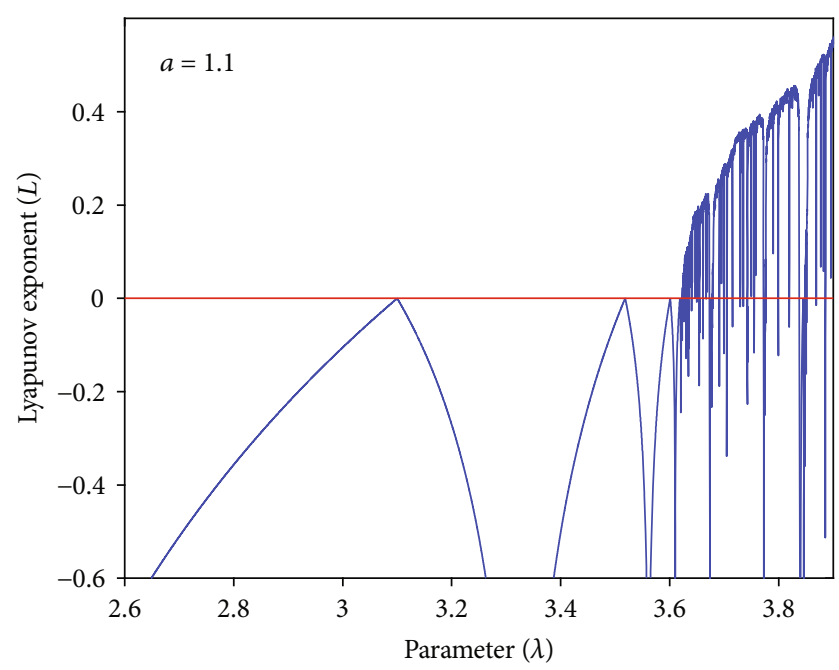

(f)

FIgURe 4: Lyapunov exponents for $a=0.1, a=0.2, a=0.3, a=0.9, a=1$, and $a=1.1$. 
For $a=0.1,0.2,0.3,0.9,1,1.1$ and certain intervals of parameter $\lambda$, the dark regions in bifurcation diagrams (Figure 2) corresponding to positive Lyapunov exponents (Figure 4) have good agreement among them which represents the chaotic behaviour in the real dynamics $f_{\lambda, a}(x)$. Further, for certain ranges, the bifurcation diagrams have white regions corresponding to negative values of Lyapunov exponents which present that the nonchaotic regions come up temporarily into chaotic regions.

\section{Conclusion}

In this article, the dynamical properties of a newly proposed two-parameter family of transcendental functions in one dimension associated with logarithmic map have been presented and simulated. The real fixed points with their nature have been theoretically found although the periodic points have been numerically simulated. By periodic-doubling as well as by period-three window in bifurcation diagrams, the presence of chaotic behaviour has been explored. Chaos is recognized by calculating positive Lyapunov exponents. As the possibility of further study in this direction, such results may extend for a family of functions having three or more parameters and moreover a family of functions having dimension more than or equal to 2 .

\section{Data Availability}

No data were used to support this study.

\section{Conflicts of Interest}

The author declares no conflict of interest.

\section{Acknowledgments}

The author gratefully acknowledges Deanship of Scientific Research, Qassim University, Saudi Arabia, for providing financial support to conduct this research under the project grant number 3666-qec-2018-1-14-S for the year 2018-2019 AD/1440-1441 AH.

\section{References}

[1] T. Kapitaniak, Chaos for Engineers: Theory Applications and Control, Springer, 2nd edition, 2000.

[2] Y. Zhou, Z. Hua, C.-M. Pun, and C. L. Philip Chen, "Cascade chaotic system with applications," IEEE Transactions on Cybernetics, vol. 45, no. 9, pp. 2001-2012, 2015.

[3] Z. Hua and Y. Zhou, "One-dimensional nonlinear model for producing chaos," IEEE Transactions on Circuits and Systems I: Regular Papers, vol. 65, no. 1, pp. 235-246, 2018.

[4] L. D. Tambasco, D. M. Harris, A. U. Oza, R. R. Rosales, and J. W. M. Bush, "The onset of chaos in orbital pilot-wave dynamics," Chaos, vol. 26, no. 10, p. 103107, 2016.

[5] J. M. T. Thompson, "Chaos, fractals and their applications," International Journal of Bifurcation and Chaos, vol. 26, no. 13, article 1630035, 2016.

[6] J. Awrejcewicz, A. V. Krysko, I. E. Kutepov, N. A. Zagniboroda, V. Dobriyan, and V. A. Krysko, "Chaotic dynamics of flexible Euler Bernoulli beams," Chaos: An Interdisciplinary Journal of Nonlinear Science, vol. 23, no. 4, article 043130, 2013.

[7] S. Khajanchi, M. Perc, and D. Ghosh, "The influence of time delay in a chaotic cancer model," Chaos, vol. 28, no. 10, article 103101, 2018.

[8] A. Naanaa, "Fast chaotic optimization algorithm based on spatiotemporal maps for global optimization," Applied Mathematics and Computation, vol. 269, pp. 402-411, 2015.

[9] L. Dobrescu, M. Neamtu, and D. Opris, "Bifurcation and chaos analysis in a discrete-delay dynamic model for a stock market," International Journal of Bifurcation and Chaos, vol. 23, no. 9, article 1350155, 2013.

[10] M. Abdelmoula and B. Robert, "Bifurcations and chaos in a photovoltaic plant," International Journal of Bifurcation and Chaos, vol. 29, no. 8, article 1950102, 2019.

[11] I. Sushko, L. Gardini, and K. Matsuyama, "Dynamics of a generalized fashion cycle model," Chaos, Solitons and Fractals, vol. 126, pp. 135-147, 2019.

[12] A. el Aroudi, L. Benadero, E. Ponce, C. Olalla, F. Torres, and L. Martinez-Salamero, "Nonlinear dynamic modeling and analysis of self-oscillating $\mathrm{H}$-bridge parallel resonant converter under zero current switching control: unveiling coexistence of attractors," IEEE Transactions on Circuits and Systems I, vol. 66, no. 4, pp. 1657-1667, 2019.

[13] E. Ponce, L. Benadero, and A. El Aroudi, "Bifurcation analysis in a self-oscillatingseries resonant converter," in Power Systems \& Smart Energies, pp. 15-28, De Gruyter, 2020.

[14] W. Gao, Y. Zhang, J. L. G. Guirao, and Y. Chen, "A discrete dynamics approach to sparse calculation and applied in ontology science," Journal of Difference Equations and Applications, vol. 25, no. 9-10, pp. 1239-1254, 2019.

[15] J. Cavro, "Recurrence in nonautonomous dynamical systems," Journal of Difference Equations and Applications, vol. 25, no. 910, pp. 1404-1411, 2019.

[16] G. Feichtinger, A. Prskawetz, A. Seidl, C. Simon, and S. Wrzaczek, "A bifurcation analysis of gender equality and fertility," Journal of Evolutionary Economics, vol. 27, no. 5, pp. 1221-1243, 2017.

[17] F. Grassetti and G. Hunanyan, "On the economic growth theory with Kadiyala production function," Communications in Nonlinear Science and Numerical Simulation, vol. 58, pp. 220-232, 2018.

[18] M. Guerrazzi and M. Sodini, "Efficiency-wage competition and nonlinear dynamics," Communications in Nonlinear Science and Numerical Simulation, vol. 58, pp. 62-77, 2018.

[19] K. T. Alligood, T. D. Sauer, and J. A. Yorke, Chaos: An Introduction to Dynamical Systems, Springer, 1997.

[20] L. A. Bunimovich and L. V. Vela-Arevalo, "Some new surprises in chaos," Chaos, vol. 25, no. 9, article 097614, 2015.

[21] M. Lakshmanan and S. Rajaseekar, Nonlinear Dynamics: Integrability, Chaos and Patterns, Springer, 2003.

[22] P. Manneville, Instabilities, Chaos and Turbulence: An Introduction to Nonlinear Dynamics and Complex Systems, World Scientific Publishing Co., 2004.

[23] T. K. Chakra and T. Nayak, "Iteration of the translated tangent," Bulletin of the Malaysian Mathematical Sciences Society, vol. 42, no. 5, pp. 1993-2008, 2019.

[24] T. Nayak and M. G. P. Prasad, "Julia sets of Joukowskiexponential maps," Complex Analysis and Operator Theory, vol. 8, no. 5, pp. 1061-1076, 2014. 
[25] M. Sajid and G. P. Kapoor, "Chaos in dynamics of a family of transcendental mero-morphic functions," Journal of Nonlinear Analysis and Application, vol. 2017, no. 1, pp. 1-11, 2017.

[26] M. Sajid and G. P. Kapoor, "Dynamics of transcendental meromorphic functions $\left(\left(z+\mu_{0}\right) /\left(z+\mu_{0}+4\right)\right) e^{z}$ having rational Schwarzian derivatives," Dynamical Systems, vol. 22, no. 3, pp. 323-337, 2007.

[27] S. Sharifi, M. Salimi, S. Siegmund, and T. Lotfi, "A new class of optimal four-point methods with convergence order 16 for solving nonlinear equations," Mathematics and Computers in Simulation, vol. 119, pp. 69-90, 2016.

[28] R. J. Brown, A Modern Introduction to Dynamical Systems, Oxford University Press, UK, 2018.

[29] A. Pikovsky and A. Politi, Lyapunov Exponents: A Tool to Explore Complex Dynamics, Cambridge University Press, 2016.

[30] D. Stauffer, H. E. Stanley, and A. Lesne, From Newton to Mandelbrot: A Primer in Theoretical Physics, Springer, 3rd edition, 2017.

[31] Y. Guo and A. C. J. Luo, "Routes of periodic motions to chaos in a periodically forced pendulum," International Journal of Dynamics and Control, vol. 5, no. 3, pp. 551-569, 2017.

[32] S. Manjunath, A. Podapati, and G. Raina, "Stability, convergence, limit cycles and chaos in some models of population dynamics," Nonlinear Dynamics, vol. 87, no. 4, pp. 25772595, 2017.

[33] L. Gardini, V. Manosa, and I. Sushko, "A route to chaos in the Boros-Moll map," International Journal of Bifurcation and Chaos, vol. 29, no. 4, article 1930009, 2019.

[34] F. Kangalgil and S. Isik, "Controlling chaos and NeimarkSacker bifurcation in a discrete-time predator-prey system," Hacettepe Journal of Mathematics and Statistics, pp. 1-16, 2020.

[35] L. Gardini, R. Makrooni, and I. Sushko, "Cascades of alternating smooth bifurcations and border collision bifurcations with singularity in a family of discontinuous linear power maps," Discrete and Continuous Dynamical Systems Series B, vol. 23, no. 2, pp. 701-729, 2018.

[36] T. Onozaki, "One-dimensional nonlinear cobweb model," in Nonlinearity, Bounded Rationality and Heterogeneity, pp. 2577, Springer, 2018.

[37] M. Ozer, A. Cenys, Y. Polatoglu et al., "Bifurcations of Fibonacci generating functions," Chaos, Solitons \& Fractals, vol. 33, no. 4, pp. 1240-1247, 2007.

[38] R. E. de Carvalho and E. D. Leonel, "Squared sine logistic map," Physica A, vol. 463, pp. 37-44, 2016.

[39] M. Sajid and A. S. Alsuwaiyan, "Chaotic behavior in the real dynamics of a one parameter family of functions," International Journal of Applied Science and Engineering, vol. 12, no. 4, pp. 283-301, 2014.

[40] D. Lim, "Fixed points and dynamics on generating function of Genocchi numbers," Journal of Nonlinear Sciences and Applications, vol. 9, no. 3, pp. 933-939, 2016.

[41] A. A. Magrenan, "A new tool to study real dynamics: the convergence plane," Applied Mathematics and Computation, vol. 248, pp. 215-224, 2014.

[42] M. Sajid, "Real fixed points and dynamics of one parameter family of function $\left(b^{x}-1\right) / x$," Journal of the Association of Arab Universities for Basic and Applied Sciences, vol. 21, no. 1, pp. 92-95, 2018.
[43] M. Sajid, "Bifurcation and chaos in real dynamics of a two-parameter family arising from generating function of generalized Apostol-type polynomials," Mathematical and Computational Applications, vol. 23, no. 1, p. 7, 2018.

[44] M. Sajid, "Chaotic behavior in real dynamics and singular values of family of generalized generating function of Apostol Genocchi numbers," Journal of Mathematics and Computer Science, vol. 19, no. 1, pp. 41-50, 2019.

[45] W. Znegui, H. Gritli, and S. Belghith, "Design of an explicit expression of the Poincare map for the passive dynamic walking of the compass-gait biped model," Chaos, Solitons and Fractals, vol. 130, article 09436, p. 109436, 2020.

[46] H. Gritli and S. Belghith, "Walking dynamics of the passive compass-gait model under OGY-based state-feedback control: rise of the Neimark Sacker bifurcation," Chaos, Solitons and Fractals, vol. 110, pp. 158-168, 2018.

[47] H. Gritli and S. Belghith, "Walking dynamics of the passive compass-gait model under OGY-based control: emergence of bifurcations and chaos," Communications in Nonlinear Science and Numerical Simulation, vol. 47, pp. 308-327, 2017.

[48] H. Gritli and S. Belghith, "Walking dynamics of the passive compass-gait model under OGY-based state-feedback control: analysis of local bifurcations via the hybrid Poincaré map," Chaos, Solitons and Fractals, vol. 98, pp. 72-87, 2017.

[49] H. Gritli and S. Belghith, "Computation of the Lyapunov exponents in the compass-gait model under OGY control via a hybrid Poincaré map," Chaos, Solitons and Fractals, vol. 81, pp. 172-183, 2015.

[50] D. Gulick, Encounters with Chaos and Fractals, Chapman and Hall/CRC, 2nd edition, 2012. 